KCL-MTH-03-13

ITP-UU-03-11

September 12, 2018

\title{
The deformed M2-brane
}

\author{
P.S. Howe ${ }^{1}$, S.F. Kerstan ${ }^{1}$, U. Lindström ${ }^{2}$ and D. Tsimpis ${ }^{1}$ \\ 1 Department of Mathematics, King's College, London, UK \\ 2 Department of Theoretical Physics Uppsala University, Sweden
}

\begin{abstract}
The superembedding formalism is used to study correction terms to the dynamics of the M2 brane in a flat background. This is done by deforming the standard embedding constraint. It is shown rigorously that the first such correction occurs at dimension four. Cohomological techniques are used to determine this correction explicitly. The action is derived to quadratic order in fermions, and the modified $\kappa$-symmetry transformations are given.
\end{abstract}




\section{Introduction}

An interesting problem in string/M theory is to consider higher-order corrections to the effective dynamics of various branes. A number of results have been obtained in the purely bosonic sector, particularly for D-branes, $[1,2,3,4,5,6,7] .{ }^{1}$ Some of these have been supersymmetrised, for example the $\partial^{4} F^{4}$ and other terms in D-brane actions [10,11], but it has so far proven difficult to obtain Lagrangians or equations of motion with fully-fledged kappa-symmetry. Some kappasymmetric results have been obtained for higher-derivative terms for superparticles $[12,13,14]$ and superstrings [15], for coincident D0-branes in [16], and for branes in lower-dimensional spacetimes in $[18,19]$. The last two papers made use of the superembedding formalism [20, 21] which offers a systematic way of incorporating kappa-symmetry whilst maintaining manifest target space supersymmetry. In this paper we shall examine the leading correction terms for the M2-brane using this formalism. We shall derive the action up to terms quadratic in the fermions as well as the modified kappa-symmetry transformations.

The two examples studied in $[18,19]$, namely a membrane in four dimensions and a set of coincident membranes in three dimensions, were relatively easy to analyse because the worldvolume multiplets in both cases are off-shell. This means that the standard superembedding constraints do not need to be altered - one only needs to find higher-order Lagrangians which can be constructed using standard methods. However, for the $1 / 2$ BPS branes in string theory and M-theory the worldvolume multiplets are maximally supersymmetric matter or gauge multiplets and it is not known how to find auxiliary fields for these or even if they exist. Indeed, the standard superembedding constraint leads directly to the equations of motion of the brane in most cases [22, 23]. This means that we need to find new techniques to discuss higher-derivative interactions for these branes. The purpose of the present paper is to show how this can be carried out in a perturbative fashion for the case of the M2-brane. The basic idea is to deform the superembedding constraint in such a way that the field content of the brane theory is unchanged. The torsion identities, which we describe in detail below, then impose consistency requirements on the deformation which can be analysed order by order in a parameter $\ell$ which has the dimensions of length and which could be the Planck length or the string length $\sqrt{\alpha^{\prime}}$. In fact, what we end up with is a cohomology problem which is similar in spirit to the cohomological problems encountered in the analysis of higher-order corrections to maximally supersymmetric field theories including supergravity $[24,25,26,27,28]$. However, the cohomology problem considered here is manifestly covariant with respect to target space supersymmetry and local worldvolume supersymmetry ( $\kappa$-symmetry); if we were to work in the static gauge the formalism would automatically have " $N=2$ " worldvolume supersymmetry (actually $d=3, N=16$ ) non-linearly realised.

We shall limit ourselves to the case of a flat target superspace in this paper for simplicity, although there will also be corrections involving the target space curvature. We show rigorously that supersymmetry does not allow any deformations with three or fewer powers of $\ell$, a result which is to be expected in view of the results that have been obtained for D-branes. We then analyse in some detail the lowest-order non-trivial deformation at $\ell^{4}$. This turns out to

\footnotetext{
${ }^{1}$ For some earlier work on higher derivative corrections see $[8,9]$.
} 
be rather complicated and involves up to seven powers in fields. We show that there is a unique deformation with at most three fields. We believe that it is unlikely that there are other independent deformations, because of the number of constraints that have to be satisfied, but we have not completed the proof of this assertion. Knowledge of the cubic terms in the deformation is sufficient to determine the modified Lagrangian to quadratic order in fermions, and thus to determine the full purely bosonic part of the action at order $\ell^{4}$. The result is, as expected, a Lagrangian which is quartic in the extrinsic curvature.

The organisation of the paper is as follows. In the next section we briefly review the superembedding formalism and the relation of worldvolume supersymmetry to $\kappa$-symmetry. In section three we solve the torsion identities for the membrane to first order in the deformation field $\psi$ and summarise the lowest order results which give the standard dynamics. In section four we introduce the relevant spinorial cohomology groups for deformed superembeddings. In section five we discuss the constraints on the deformation and solve the relevant cohomology problem up to order $\ell^{4}$ in section six. In section seven we construct the action, up to terms quadratic in the fermions, and in section eight we discuss the modified $\kappa$-symmetry transformations.

\section{Superembeddings}

The superembedding formalism was pioneered in the context of superparticles in three and four dimensions by Sorokin, Tkach, Volkov and Zheltukhin [20], and has been applied by these and other authors to various other branes; for a review see [21]. ${ }^{2}$ In [22] it was shown that the formalism can be applied to arbitrary branes including those with various types of worldvolume gauge fields, and it was then used to construct the full non-linear equations of motion of the M-theory 5-brane in an arbitrary supergravity background [23].

We consider a superembedding $f: M \rightarrow \underline{M}$, where $M$ is the worldvolume of the brane and $\underline{M}$ is the target space. Our index conventions are as follows: coordinate indices are taken from the middle of the alphabet with capitals for all, Latin for bosonic and Greek for fermionic, $M=$ $(m, \mu)$, tangent space indices are taken in a similar fashion from the beginning of the alphabet so that $A=(a, \alpha)$. We denote the coordinates of $M$ by $z^{M}=\left(x^{m}, \theta^{\mu}\right)$. The distinguished tangent space bases are related to coordinate bases by means of the supervielbein, $E_{M}{ }^{A}$, and its inverse $E_{A}{ }^{M}$. We use exactly the same notation for the target space but with all of the indices underlined. Indices for the normal bundle are denoted by primes, so that $A^{\prime}=\left(a^{\prime}, \alpha^{\prime}\right)$. We shall occasionally group together tangent and normal indices and denote them by a bar, $\bar{a}=\left(a, a^{\prime}\right)$ etc.

The embedding matrix is the derivative of $f$ referred to the preferred tangent frames, thus

$$
E_{A} \underline{A}:=E_{A}^{M} \partial_{M} z^{\underline{M}} E_{\underline{M}}^{\underline{A}}
$$

In addition, we can specify a basis $\left\{E_{A^{\prime}}\right\}$ for the normal bundle in terms of the target space basis by means of the normal matrix $E_{A^{\prime}} \underline{A}$.

\footnotetext{
${ }^{2}$ For an earlier attempt to use source and target superspaces, see, e.g., [29].
} 
The basic embedding condition is

$$
E_{\alpha} \underline{a}=0
$$

Geometrically this states that the odd tangent space of the brane is a subspace of the odd tangent space of the target superspace at any point on the brane. To see the content of this constraint we can consider a linearised embedding in a flat target space in the static gauge. This gauge is specified by identifying the coordinates of the brane with a subset of the coordinates of the worldvolume, so that

$$
\begin{aligned}
& x^{\underline{a}}=\left\{\begin{array}{l}
x^{a} \\
x^{a^{\prime}}(x, \theta)
\end{array}\right. \\
& \theta^{\underline{\alpha}}=\left\{\begin{array}{l}
\theta^{\alpha} \\
\theta^{\alpha^{\prime}}(x, \theta)
\end{array}\right.
\end{aligned}
$$

Since

$$
E^{\underline{a}}=d x^{\underline{a}}-\frac{i}{2} d \theta^{\underline{\alpha}}\left(\Gamma^{\underline{a}}\right)_{\underline{\alpha} \underline{\beta}} \theta^{\underline{\beta}}
$$

in flat space, it is easy to see, to first order in the transverse fields, that the embedding condition implies that

$$
D_{\alpha} X^{a^{\prime}}=i\left(\Gamma^{a^{\prime}}\right)_{\alpha \beta^{\prime}} \theta^{\beta^{\prime}}
$$

where

$$
X^{a^{\prime}}=x^{a^{\prime}}+\frac{i}{2} \theta^{\alpha}\left(\Gamma^{a^{\prime}}\right)_{\alpha \beta^{\prime}} \theta^{\beta^{\prime}}
$$

From this equation the nature of the worldvolume multiplet specified by the embedding condition can be determined. Depending on the dimensions involved, this multiplet can be one of three types: (i) on-shell, i.e. the multiplet contains only physical fields and these fields satisfy equations of motion, (ii) Lagrangian off-shell, meaning that the multiplet also contains auxiliary fields (in most cases), and that the equations of motion of the physical fields are not satisfied, although they can be derived from a superfield action, and (iii) underconstrained, which means that further constraints are required to obtain multiplets of one of the first two types. For thirty-two target space supersymmetries, the multiplets are always of type (i) or of type (iii), whereas for sixteen or fewer supersymmetries all three types of multiplet can occur, with type (iii) arising typically for cases of low even codimension.

In this paper we shall be concerned with the membrane in $D=11$ superspace, i.e. the M2brane. This has been discussed as a superembedding in [30]. The worldvolume is $d=3, N=8$ superspace, and the worldvolume multiplet is an on-shell scalar multiplet (type (i)). This can be 
seen from (7). The leading component of the superfield $X^{a^{\prime}}$ describes eight scalar fields on the worldvolume, while the leading component of $\theta^{\alpha^{\prime}}$ describes eight spin one-half fields. Equation (7) implies that there are no further independent component fields and that the scalars and spinors satisfy the usual equations of motion for free massless fields.

In order to determine the consequences of the superembedding condition in the non-linear case, and to find the induced supergeometry on the brane, one uses the torsion equation which is the pull-back of the equation defining the target space torsion two-form. This reads

$$
2 \nabla_{[A} E_{B]}^{\underline{C}}+T_{A B}^{C} E_{C} \underline{\underline{C}}=(-1)^{A(B+\underline{B})} E_{B} \underline{\underline{B}} E_{A^{\underline{A}}} T_{\underline{A B}}^{\underline{C}}
$$

In this equation the covariant derivative acts on both worldvolume and target space tensor indices. The latter are taken care of by the pull-back of the target space connection, while the worldvolume connection can be chosen in a variety of different ways. One also has to parametrise the embedding matrix. Having done all this, one can work through the torsion equation starting at dimension zero. In this way the consequences of the embedding condition (2) can be worked out in a systematic and covariant fashion.

The equations of the component or Green-Schwarz formalism can be obtained by taking the leading $(\theta=0)$ components of the various equations that describe the brane multiplet. A key feature of the formalism is that these component equations are guaranteed to be $\kappa$-symmetric because $\kappa$-symmetry can be identified with the leading term in a worldvolume local supersymmetry transformation. We recall briefly how this works [20]. Let $v^{M}$ be a worldvolume vector field generating an infinitesimal diffeomorphism. If we write the superembedding in local coordinates as $f \underline{M}=z \underline{M}(X)$, then the effect of such a transformation on $\underline{z}(z)$ is

$$
\delta z^{\underline{M}}=v^{M} \partial_{M} z^{\underline{M}}
$$

If we express this in the preferred bases we find

$$
\delta z^{\underline{A}}:=\delta z^{\underline{M}} E_{\underline{M}} \underline{A}=v^{A} E_{A} \underline{A}
$$

Now if we take an odd worldvolume diffeomorphism, $v^{a}=0$, and use the embedding condition (2) we get

$$
\begin{aligned}
\delta z^{\underline{a}} & =0 \\
\delta z^{\underline{\alpha}} & =v^{\alpha} E_{\alpha} \underline{\alpha}
\end{aligned}
$$

This can be brought to the more usual $\kappa$-symmetric form if we define

$$
\kappa^{\underline{\alpha}}:=v^{\alpha} E_{\alpha} \underline{\alpha}
$$

and note that it satisfies 


$$
\kappa^{\underline{\alpha}}=\kappa^{\underline{\beta}} P_{\underline{\beta}}^{\underline{\alpha}}:=\frac{1}{2} \kappa^{\underline{\beta}}(1+\Gamma)_{\underline{\beta}}^{\underline{\alpha}}
$$

where $P$ is the projection operator onto the worldvolume subspace of the odd tangent space of the target superspace. We can always write $P=1 / 2(1+\Gamma)$, and so $\Gamma$ is computable in terms of the embedding matrix. Substituting this into (12) we recover the normal form of $\kappa$ symmetry transformations. (Strictly, we should evaluate this equation at $\theta=0$ to get the correct component form.) The explicit form of $P$ is

$$
P_{\underline{\alpha}}^{\underline{\beta}}=\left(E^{-1}\right)_{\underline{\alpha}}^{\gamma} E_{\gamma} \underline{\beta}
$$

where the inverse is taken in the fermionic tangent space (of $\underline{M}$ ).

For the case of the supermembrane in $D=11$, this procedure yields the equations of motion found in [31]. To find higher derivative corrections to these equations it follows that we shall need to amend the basic embedding condition (2). We note that a simple consequence of this is that the $\kappa$-symmetry transformations will no longer have the standard characteristic form for which $\delta z^{\underline{a}}=0$.

\section{The torsion identities}

In this section we shall study the torsion identities in the case that the embedding condition is relaxed. We shall take the target space to be flat so that the only non-vanishing component of the target space torsion is

$$
T_{\underline{\alpha \beta}} \underline{a}=-i\left(\Gamma^{\underline{a}}\right)_{\underline{\alpha \beta}},
$$

We can parametrise the embedding matrix as follows:

$$
\begin{array}{ll}
E_{\alpha} \underline{\underline{a}}=\psi_{\alpha}^{a^{\prime}} u_{a^{\prime}} \underline{a} & E_{\alpha} \underline{\underline{\alpha}}=u_{\alpha} \underline{\underline{\alpha}}+h_{\alpha^{\alpha^{\prime}}} u_{a^{\prime}} \underline{\underline{\alpha}} \\
E_{a} \underline{\underline{a}}=u_{a} \underline{\underline{a}}, & E_{a} \underline{\underline{\alpha}}=\Lambda_{a}{ }^{\alpha^{\prime}} u_{\alpha^{\prime}} \underline{\underline{\alpha}}
\end{array}
$$

while the normal matrix can be chosen to have the form

$$
\begin{array}{ll}
E_{\alpha^{\prime}} \underline{a}=0, & E_{\alpha^{\prime}} \underline{\underline{\alpha}}=u_{\alpha^{\prime}} \underline{\underline{\alpha}} \\
E_{a^{\prime}} \underline{a}=u_{a^{\prime}} \underline{a}, & E_{a^{\prime}} \underline{\underline{\alpha}}=0
\end{array}
$$

Here, the $32 \times 32$ matrix $u_{\bar{\alpha}} \underline{\alpha}:=\left(u_{\alpha} \underline{\alpha}, u_{\alpha^{\prime}} \underline{\alpha}\right)$ is an element of $\operatorname{Spin}(1,10)$ while the matrix $u_{\bar{a}} \underline{a}:=\left(u_{a} \underline{a}, u_{a^{\prime}} \underline{a}\right)$ is the corresponding element of $S O(1,10)$. The dimensions, in units of mass, of the various components of the embedding matrix are given by 


$$
\left[E_{\alpha} \underline{\underline{\alpha}}\right]=\left[E_{a} \underline{a}\right]=0, \quad\left[E_{\alpha} \underline{\underline{a}}\right]=-\left[E_{a} \underline{\underline{\alpha}}\right]=-\frac{1}{2}
$$

and similarly for the normal matrix. We can always bring these matrices into the above forms by a suitable choice of the bases of the even and odd tangent spaces on the worldvolume. We can now plug this form of the embedding matrix into the torsion identity

$$
\nabla_{A} E_{B} \underline{C}-(-)^{A B} \nabla_{B} E_{A} \underline{C}+T_{A B}^{C} E_{C} \underline{C}=(-)^{A(B+\underline{B})} E_{B} \underline{\underline{B}} E_{A} \underline{\underline{A}} T_{\underline{A B}} \underline{C}
$$

and work out the consequences. As we noted earlier, the covariant derivative here acts on both target space and worldvolume indices, but since the target space is flat we only need a worldvolume connection. This can be specified either by imposing some constraints on the worldvolume torsion tensor or by choosing a connection which is natural in the superembedding context. We shall use the latter. Given the matrix $u$ we can define the set of one-forms

$$
X_{A}:=\left(\nabla_{A} u\right) u^{-1}
$$

If we set

$$
X_{A, b}{ }^{c}=X_{A, b^{\prime}}{ }^{\prime}=0
$$

then we fix connections for the tangent and normal bundles in a standard fashion. Note that

$$
X_{A, \bar{\beta}} \bar{\gamma}=\frac{1}{4}\left(\Gamma^{\bar{b}} \bar{c}\right)_{\bar{\beta}} \bar{\gamma} X_{A, \bar{b} \bar{c}}
$$

The torsion identities yield the following results for a flat target superspace:

$\operatorname{dim} 0$

$$
\begin{aligned}
& T_{\alpha \beta}^{c}=-i\left(\Gamma^{c}\right)_{\alpha \beta}+2 \psi_{(\alpha}{ }^{d^{\prime}} X_{\beta), d^{\prime}}{ }^{c} \\
& \nabla_{(\alpha} \psi_{\beta)}{ }^{c^{\prime}}=-i h_{(\alpha} \gamma^{\prime}\left(\Gamma^{c^{\prime}}\right)_{\beta) \gamma^{\prime}}+\left(-T_{\alpha \beta} \psi_{\gamma}{ }^{c^{\prime}}\right)
\end{aligned}
$$

$\operatorname{dim} 1 / 2$

$$
\begin{aligned}
T_{\alpha b}{ }^{c} & =\psi_{\alpha}{ }^{d^{\prime}} X_{b, d^{\prime}}{ }^{c}+i \Lambda_{b}{ }^{\prime} h_{\alpha}{ }^{\gamma^{\prime}}\left(\Gamma^{c}\right)_{\beta^{\prime} \gamma^{\prime}} \\
X_{\alpha b} c^{\prime} & =\nabla_{b} \psi_{\alpha}{ }^{c^{\prime}}+i \Lambda_{b}{ }^{\prime}\left(\Gamma^{c^{\prime}}\right)_{\alpha \beta^{\prime}} \\
T_{\alpha \beta}{ }^{\gamma} & =-2 h_{(\alpha}{ }^{\delta^{\prime}} X_{\beta), \delta^{\prime}}{ }^{\gamma} \\
2 \nabla_{(\alpha} h_{\beta)}{ }^{\gamma^{\prime}} & =-2 X_{(\alpha, \beta)}{ }^{\gamma^{\prime}}-T_{\alpha \beta}{ }^{c} \Lambda_{c}{ }^{\gamma^{\prime}}-\left(T_{\alpha \beta}{ }^{\delta} h_{\delta} \gamma^{\prime}\right)
\end{aligned}
$$

$\operatorname{dim} 1$ 


$$
\begin{aligned}
T_{a b}{ }^{c} & =i \Lambda_{a}{ }^{\alpha^{\prime}} \Lambda_{b}{ }^{\beta^{\prime}}\left(\Gamma^{c}\right)_{\alpha^{\prime} \beta^{\prime}} \\
2 X_{[a b]} c^{\prime} & =-T_{a b}{ }^{\gamma} \psi_{\gamma}^{\gamma^{\prime}} \\
T_{a \beta}{ }^{\gamma} & =-\Lambda_{a}{ }^{\delta^{\prime}} X_{\beta, \delta^{\prime}}{ }^{\gamma}-h_{\beta}{ }^{\delta^{\prime}} X_{a, \delta^{\prime}} \gamma \\
X_{a, \beta} \gamma^{\prime} & =\nabla_{\beta} \Lambda_{a}{ }^{\gamma^{\prime}}-\nabla_{a} h_{\beta} \gamma^{\gamma^{\prime}}
\end{aligned}
$$

$\operatorname{dim} 3 / 2$

$$
\begin{aligned}
T_{a b}{ }^{\gamma} & =2 \Lambda_{[a}^{\delta^{\prime}} X_{b], \delta^{\prime}}{ }^{\gamma} \\
2 \nabla_{[a} \Lambda_{b]} \gamma^{\prime} & =-T_{a b}{ }^{c} \Lambda_{c}{ }^{\gamma^{\prime}}-T_{a b}{ }^{\delta} h_{\delta}{ }^{\prime}
\end{aligned}
$$

The terms in brackets will be irrelevant in what follows because they will turn out to be zero at first order in the deformation parameter as we shall shortly see.

\subsection{Zeroth order}

In the zeroth order theory the standard embedding condition $E_{\alpha} \underline{a}=0$ holds, so that $\psi_{\alpha}{ }^{\prime}=0$. If we substitute this into the above equations we find that $h_{\alpha}{ }^{\beta^{\prime}}=0$ while the induced torsion is

$$
T_{\alpha \beta}^{c}=-i\left(\Gamma^{c}\right)_{\alpha \beta}
$$

at dimension zero and vanishes at dimension one-half. The dimension one and three-halves components are given by the corresponding equations above.

For $X$ we find, at dimension one-half,

$$
\begin{aligned}
X_{\alpha, b}{ }^{c^{\prime}} & =i \Lambda_{b}{ }^{\beta^{\prime}}\left(\Gamma^{c^{\prime}}\right)_{\alpha \beta^{\prime}} \\
2 X_{(\alpha \beta)}{ }^{\gamma^{\prime}} & =i\left(\Gamma^{c}\right)_{\alpha \beta} \Lambda_{c}{ }^{\gamma^{\prime}}
\end{aligned}
$$

It is easy to show that these equations imply that

$$
\left(\Gamma^{a}\right)_{\alpha^{\prime} \beta^{\prime}} \Lambda_{a}^{\beta^{\prime}}=0
$$

In the linearised theory $\Lambda_{a}{ }^{\beta^{\prime}} \sim \partial_{a} \theta^{\beta^{\prime}}$, so that we can identify (41) as the equation of motion of the fermion field in the worldvolume multiplet. At dimension one we have

$$
\begin{aligned}
& X_{[a b]} c^{c^{\prime}}=0 \\
& X_{a, \beta} \gamma^{\gamma^{\prime}}=\nabla_{\beta} \Lambda_{a} \gamma^{\prime}
\end{aligned}
$$


Using the fermion equation of motion we find the scalar equation of motion

$$
\eta^{a b} X_{a, b}{ }^{\prime}=0
$$

Indeed, in the linearised theory, $X_{a b}{ }^{c^{\prime}} \sim \partial_{a} \partial_{b} X^{c^{\prime}}$, so we get the massless Klein-Gordon equation. Finally, a short calculation gives the supersymmetry variation of $X_{a b} c^{\prime}$ :

$$
\nabla_{\alpha i} X_{b, c}{ }^{d^{\prime}}=-i\left(\sigma^{d^{\prime}} \nabla_{(b} \Lambda_{c)}\right)_{\alpha i}+\frac{1}{2}\left(\Lambda_{b} \gamma^{e} \otimes \tilde{\sigma}^{f^{\prime} d^{\prime}} \Lambda_{c}\right)\left(\sigma_{f^{\prime}} \Lambda_{e}\right)_{\alpha i}
$$

\subsection{First order}

A first order deformation of the theory will involve the presence of a non-vanishing $\psi$ field which we can take to be of the form of a dimensionful parameter $\beta$, say, multiplied by a function of the physical fields $\Lambda_{a}^{\beta^{\prime}}$ and $X_{a b} c^{\prime}$ as well as derivatives. We shall write this schematically as

$$
\psi=\beta f(\Lambda, X, \partial)
$$

Furthermore, $\psi$ is subject to the constraint

$$
\nabla_{(\alpha} \psi_{\beta)}{ }^{c^{\prime}}=-i h_{(\alpha}^{\gamma^{\prime}}\left(\Gamma^{c^{\prime}}\right)_{\beta) \gamma^{\prime}}
$$

Note that, since $\psi, h$ and $T_{\alpha \beta}{ }^{\gamma}$ are all of order $\beta$ we are allowed to drop the $T_{\alpha \beta}{ }^{\gamma} \psi_{\gamma}{ }^{\prime}$ term from (27). The problem is then to analyse equation (47). This can be done systematically in powers of the length parameter $\ell$. Note that, given an explicit expression for $\psi$ in terms of the physical fields, equation (47) allows us to solve for $h$.

\section{Spinorial cohomology for branes}

The notion of spinorial cohomology $[24,26]$ is useful for studying both the space of physical fields in certain supersymmetric theories and also for studying deformations of the equations of motion. In the simplest case one studies spinorial $p$-forms, i.e. totally symmetric $p$-spinors which are $\gamma$-traceless, together with a differential operator which is obtained by acting with $D_{\alpha}$ followed by symmetrisation and removal of the $\gamma$-trace. This defines a cohomology which is isomorphic to pure spinor cohomology $[32,33]$. The relevance of the latter to theories in ten and eleven dimensions is explained by the fact that the equations of motion can be interpreted in terms of pure spinor integrability $[34,35]$. The formalism has been applied to $D=10$ Yang-Mills theory and supergravities in ten and eleven dimensions [24, 25, 26, 27, 28]. Moreover, one can also consider vector-valued spinorial cohomology [26]. For branes, the appropriate cohomology is a variant on the latter.

Since we are interested in first-order deformations, it is sufficient to work on a worldvolume whose induced supergeometry is given by the zeroth order theory. We then consider the spaces 
$\Omega_{B^{\prime}}^{p}$ and $\Omega_{F^{\prime}}^{p}$. The objects in these spaces are spinorial $p$-forms on $M$ which take their values either in the even normal bundle $B^{\prime}$ or the odd normal bundle $F^{\prime}$. There is a natural map $\Gamma: \Omega_{F^{\prime}}^{p-1} \rightarrow \Omega_{B^{\prime}}^{p}$ given by

$$
h_{\alpha_{1} \ldots \alpha_{p-1}}^{\gamma^{\prime}} \mapsto-i h_{\left(\alpha_{1} \ldots \alpha_{p-1}\right.} \gamma^{\prime}\left(\Gamma^{c^{\prime}}\right)_{\left.\alpha_{b}\right) \gamma^{\prime}}
$$

We can therefore form the quotient space $\hat{\Omega}_{B^{\prime}}^{p}:=\Omega_{B^{\prime}}^{p} / \Gamma\left(\Omega_{F^{\prime}}^{p-1}\right)$ and construct a derivative $d_{s}$ : $\hat{\Omega}_{B^{\prime}}^{p} \rightarrow \hat{\Omega}_{B^{\prime}}^{p+1}$ which squares to zero. This derivative is defined by acting with the spinorial covariant derivative $\nabla_{\alpha}$ and symmetrising modulo equivalences of the form of equation (48). In other words, if $h \in \Omega_{B^{\prime}}^{p}$ represents an equivalence class $[h] \in \hat{\Omega}_{B^{\prime}}^{p}$, then $d_{s}[h]=[\nabla h] \in \hat{\Omega}_{B^{\prime}}^{p+1}$ where

$$
(\nabla h)_{\alpha_{1} \ldots \alpha_{p+1}} c^{\prime}:=\nabla_{\left(\alpha_{1}\right.} h_{\left.\alpha_{2} \ldots \alpha_{p+1}\right)} c^{\prime}
$$

It is easy to see that this definition is independent of the choice of representative $h$. To see that $d_{s}^{2}=0$ we observe that

$$
\nabla_{\left(\alpha_{1}\right.} \nabla_{\alpha_{2}} h_{\left.\alpha_{3} \ldots \alpha_{p+2}\right)}{ }^{c^{\prime}}=-\frac{1}{2} R_{\left(\alpha_{1} \alpha_{2}, \alpha_{3}\right.}^{\gamma} h_{\left.|\gamma| \alpha_{4} \ldots \alpha_{p+2}\right)}{ }^{c^{\prime}}+\frac{1}{2} R_{\left(\alpha_{1} \alpha_{2}, d^{\prime}\right.}^{c^{\prime}} h_{\left.\alpha_{3} \ldots \alpha_{p+2}\right)} d^{d^{\prime}}
$$

Now, from the first Bianchi identity, we have

$$
\begin{aligned}
R_{\left(\alpha_{1} \alpha_{2} \alpha_{3}\right)}^{\gamma} & =\nabla_{\left(\alpha_{1}\right.} T_{\left.\alpha_{2} \alpha_{3}\right)}^{\gamma}+T_{\left(\alpha_{1} \alpha_{2}\right.}{ }^{B} T_{\left.B \alpha_{3}\right)}{ }^{\gamma} \\
& =-i\left(\Gamma^{b}\right)_{\left(\alpha_{1} \alpha_{2}\right.} T_{\left.b \alpha_{3}\right)}^{\gamma}
\end{aligned}
$$

so that the first term on the RHS of (50) is of the form

$$
-i\left(\Gamma^{b}\right)_{\left(\alpha_{1} \alpha_{2}\right.} k_{\left.b \alpha_{3} \ldots \alpha_{p+2}\right)} c^{\prime}
$$

for some $k$. However, this can be written as

$$
-i h_{\left(\alpha_{1} \ldots \alpha_{p+1}\right.} \gamma^{\prime}\left(\Gamma^{c^{\prime}}\right)_{\left.\alpha_{p+2}\right) \gamma^{\prime}}
$$

where

$$
h_{\alpha_{1} \ldots \alpha_{p+1}}^{\gamma^{\prime}}=\left(\Gamma^{b c^{\prime}}\right)_{\left(\alpha_{1}\right.}{ }^{\prime} k_{\left.b \alpha_{2} \ldots \alpha_{p+2}\right) c^{\prime}}
$$

and so this term maps to zero in the quotient space. The second term on the RHS of (50) can be evaluated with the aid of the Gauss-Codazzi equation which can, in turn, be derived from the definition of $X_{A}$. One finds that

$$
R_{\alpha \beta c^{\prime}}{ }^{\prime}=2 X_{\left(\alpha c^{\prime}\right.}^{e} X_{\beta) e^{d^{\prime}}}
$$


Since $X_{\alpha b} c^{\prime}=i \Lambda_{b}{ }^{\beta^{\prime}}\left(\Gamma^{c^{\prime}}\right)_{\beta^{\prime} \alpha}$ we have

$$
R_{\alpha \beta c^{\prime}} d^{\prime}=2 \Lambda^{e \gamma^{\prime}} \Lambda_{e}^{\delta^{\prime}}\left(\Gamma^{c^{\prime}}\right)_{\gamma^{\prime}(\alpha}\left(\Gamma^{d^{\prime}}\right)_{\beta) \delta^{\prime}}
$$

from which it is easy to see that the second term also gives zero in the quotient space. This shows that $d_{s}^{2}=0$ so that we can define the cohomology groups $H_{B^{\prime}}^{p}:=\operatorname{ker} d_{s} \cap \hat{\Omega}_{B^{\prime}}^{p} / \operatorname{im} d_{s} \hat{\Omega}_{B^{\prime}}^{p-1}$. We claim that a first-order deformation of the dynamics of the membrane is given by an element of $H_{B^{\prime}}^{1}$ (phys), where the notation indicates that the coefficients should be given by fields constructed from the physical fields.

To see that this is the appropriate group we need to consider redefinitions. We shall find the effect of a field redefinition of the embedding coordinates

$$
z^{\underline{M}} \rightarrow z^{\underline{M}}+(\delta z)^{\underline{M}}
$$

on the field $\psi_{\alpha}{ }^{\prime}$. The transformation (57) of the embedding coordinates with the background geometry fixed may be viewed equivalently as a diffeomorphism of the background geometry with the embedding coordinates fixed. Taking the latter point of view, we find that the target-space vielbein transforms as

$$
\delta E_{\underline{M}}^{\underline{A}}=(\delta z)^{\underline{N}} T_{\underline{N M}} \underline{A}+\nabla_{\underline{M}}(\delta z) \underline{A},
$$

up to a Lorentz transformation on the flat index. The transformation of the embedding matrix reads

$$
\begin{aligned}
\delta E_{A} \underline{\underline{A}} & =E_{A}^{M} \partial_{M} Z^{\underline{M}} \delta E_{\underline{M}} \underline{A} \\
& =\nabla_{A}(\delta z)^{\underline{A}}+E_{A} \underline{\underline{C}}(\delta z)^{\underline{B}} T_{\underline{B C}} \underline{A}
\end{aligned}
$$

Setting $A=\alpha, \underline{A}=\underline{a}$ in the above and expressing $E_{\alpha} \underline{a}$ in terms of $\psi_{\alpha}{ }^{c^{\prime}}$, we obtain

$$
\delta \psi_{\alpha}{ }^{c^{\prime}}=\nabla_{\alpha}(\delta z)^{c^{\prime}}+(\delta z)^{b} X_{\alpha b}{ }^{c^{\prime}}-i(\delta z)^{\gamma^{\prime}}\left(\Gamma^{c^{\prime}}\right)_{\gamma^{\prime} \alpha}
$$

where $(\delta z)^{\bar{c}}:=(\delta z)^{\underline{c}} u_{\underline{c}}{ }^{\bar{c}}$, etc. Since $\delta z$ is of order $\beta$ we can use the zeroth order expression for $X$ and so we obtain

$$
\delta \psi_{\alpha}{ }^{c^{\prime}}=\nabla_{\alpha}(\delta z)^{c^{\prime}}-i h^{\gamma^{\prime}}\left(\Gamma^{c^{\prime}}\right)_{\gamma^{\prime} \alpha}
$$

where

$$
h^{\gamma^{\prime}}:=(\delta z)^{\gamma^{\prime}}-(\delta z)^{b} \Lambda_{b} \gamma^{\prime}
$$

Together with the fact that $\psi$ satisfies the constraint (47), this proves the contention that the first-order deformation is indeed given by an element of $H_{B^{\prime}}^{1}($ phys).

We observe that the zeroth order group $H_{B^{\prime}}^{0}$ can be interpreted as the space of physical fields, since a deformation of the form of (59) such that the left-hand side vanishes will leave the basic 
embedding constraint unchanged. Indeed, the linearised embedding equation (2) can also be viewed as defining an element of this group in flat space.

The discussion given here is pertinent to the M2-brane but can be generalised to other branes. This may require some technical modifications since the field $h_{\alpha} \gamma^{\prime}$ does not vanish at zeroth order in the presence of worldvolume gauge fields; in particular, this is the case for D-branes and the M5-brane.

\section{Constraints on $\psi$}

We now derive the constraints on $\psi_{\alpha}{ }^{a^{\prime}}$ implied by the dimension-0 torsion identity at linear order in $\beta$,

$$
\nabla_{(\alpha} \psi_{\beta)}{ }^{c^{\prime}}=-i h_{(\alpha}{ }^{\prime}\left(\Gamma^{c^{\prime}}\right)_{\beta) \gamma^{\prime}}
$$

Note that in the above we have taken into account that $T_{\alpha \beta}^{\gamma}$ is of order $\beta$ as implied by the dimension $-1 / 2$ torsion identity.

The field $\psi_{\alpha i}{ }^{a^{\prime}}$ (switching to two-step notation) transforms under the $(1) \times((0001) \otimes(1000))$ representation of $\operatorname{Spin}(1,2) \times \operatorname{Spin}(8)$. (See the appendix for representation-theoretic conventions.) Decomposing $\psi$ into irreducible representations, we find

$$
\psi_{\alpha i}{ }^{a^{\prime}} \sim(1) \times(1001) \oplus(1) \times(0010)
$$

Explicitly:

$$
\begin{aligned}
\psi_{\alpha i}{ }^{a^{\prime}} & =\Sigma_{\alpha i}{ }^{a^{\prime}} & & (1) \times(1001) \\
& +\left(\sigma^{a^{\prime}}\right)_{i j^{\prime}} \Sigma_{\alpha}^{j^{\prime}} & & (1) \times(0010)
\end{aligned}
$$

From (59) it follows that the trace part in the decomposition of $\psi_{\alpha i}^{a^{\prime}}$ above can be eliminated using field redefinitions of the embedding coordinates. We shall therefore set $\Sigma_{\alpha}^{i^{\prime}}=0$.

Similarly, the field $h_{\alpha}^{\beta^{\prime}} \rightarrow h_{\alpha i}{ }^{\beta j^{\prime}}$ (in two-step notation) transforms under the $((1) \otimes(1)) \times$ $((0001) \otimes(0010))$ representation of $\operatorname{Spin}(1,2) \times \operatorname{Spin}(8)$. Decomposing in irreducible representations, we have

$$
h_{\alpha i}{ }^{\beta j^{\prime}} \sim(0) \times(1000) \oplus(0) \times(0011) \oplus(2) \times(1000) \oplus(2) \times(0011)
$$

Explicitly

$$
\begin{aligned}
h_{\alpha i}{ }^{\beta j^{\prime}} & =\delta_{\alpha}^{\beta}\left(\sigma^{a^{\prime}}\right)_{i}^{j^{\prime}} h_{a^{\prime}} & & (0) \times(1000) \\
& +\frac{1}{6} \delta_{\alpha}^{\beta}\left(\sigma^{a^{\prime} b^{\prime} c^{\prime}}\right)_{i}^{j^{\prime}} h_{a^{\prime} b^{\prime} c^{\prime}} & & (0) \times(0011) \\
& +\left(\gamma^{a}\right)_{\alpha}^{\beta}\left(\sigma^{a^{\prime}}\right)_{i}^{j^{\prime}} h_{a a^{\prime}} & & (2) \times(1000) \\
& +\frac{1}{6}\left(\gamma^{a}\right)_{\alpha}{ }^{\beta}\left(\sigma^{a^{\prime} b^{\prime} c^{\prime}}\right)_{i}^{j^{\prime}} h_{a a^{\prime} b^{\prime} c^{\prime}} & & (2) \times(0011)
\end{aligned}
$$

In order to analyse (61) we need to decompose $\nabla_{\alpha i} \Sigma_{\beta j}{ }^{\prime}$ into irreducible representations of $\operatorname{Spin}(1,2) \times \operatorname{Spin}(8)$, (remember we have set $\Sigma_{\alpha}^{i^{\prime}}$ to zero). This field transforms under the 
$((1) \otimes(1)) \times((0001) \otimes(1001))$ representation. Decomposing into irreducible representations, we have

$$
\begin{aligned}
\nabla_{\alpha i} \Sigma_{\beta j}{ }^{a^{\prime}} & \sim(0) \times(1000) \oplus(0) \times(0011) \oplus(0) \times(1100) \\
& \oplus(2) \times(1000) \oplus(2) \times(0011) \oplus(2) \times(1002)
\end{aligned}
$$

Explicitly,

$$
\begin{array}{rlrl}
\nabla_{\alpha i} \Sigma_{\beta j}^{a^{\prime}} & =\varepsilon_{\alpha \beta} \delta_{i j} Y^{a^{\prime}}+\frac{1}{7} \varepsilon_{\alpha \beta}\left(\sigma^{a^{\prime} b^{\prime}}\right)_{i j} Y_{b^{\prime}} & (0) \times(1000) \\
& +\frac{1}{2} \varepsilon_{\alpha \beta}\left(\sigma_{b^{\prime} c^{\prime}}\right)_{i j} Y^{a^{\prime} b^{\prime} c^{\prime}}+\frac{1}{10} \varepsilon_{\alpha \beta}\left(\sigma^{a^{\prime} b^{\prime} c^{\prime} d^{\prime}}\right)_{i j} Y_{b^{\prime} c^{\prime} d^{\prime}} & (0) \times(0011) \\
& +\frac{1}{2} \varepsilon_{\alpha \beta}\left(\sigma_{b^{\prime} c^{\prime}}\right)_{i j} Y^{b^{\prime} c^{\prime} ; a^{\prime}} & & (0) \times(1100) \\
& +\frac{1}{120} \varepsilon_{\alpha \beta}\left(\sigma_{b^{\prime} c^{\prime} d^{\prime} e^{\prime}}\right)_{i j} Y^{b^{\prime} c^{\prime} d^{\prime} e^{\prime} ; a^{\prime}} & & (0) \times(1002) \\
& +\left(\gamma^{a}\right)_{\alpha \beta} \delta_{i j} Y_{a} a^{\prime}+\frac{1}{7}\left(\gamma^{a}\right)_{\alpha \beta}\left(\sigma^{a^{\prime} b^{\prime}}\right)_{i j} Y_{a b^{\prime}} & (2) \times(1000) \\
& +\frac{1}{2}\left(\gamma^{a}\right)_{\alpha \beta}\left(\sigma_{b^{\prime} c^{\prime}}\right)_{i j} Y_{a}^{a^{\prime} b^{\prime} c^{\prime}}+\frac{1}{10}\left(\gamma^{a}\right)_{\alpha \beta}\left(\sigma^{a^{\prime} b^{\prime} c^{\prime} d^{\prime}}\right)_{i j} Y_{a b^{\prime} c^{\prime} d^{\prime}} & & (2) \times(0011) \\
& +\frac{1}{2}\left(\gamma^{a}\right)_{\alpha \beta}\left(\sigma_{b^{\prime} c^{\prime}}\right)_{i j} Y_{a}^{b^{\prime} c^{\prime} ; a^{\prime}} & & (2) \times(1100) \\
& +\frac{1}{120}\left(\gamma^{a}\right)_{\alpha \beta}\left(\sigma_{b^{\prime} c^{\prime} d^{\prime} e^{\prime}}\right)_{i j} Y_{a}^{b^{\prime} c^{\prime} d^{\prime} e^{\prime} ; a^{\prime}} & & (2) \times(1002)
\end{array}
$$

The semi-colon notation here denotes "hook" representations, e.g $a^{\prime} b^{\prime} ; c^{\prime}$ denotes a traceless tensor which is antisymmetric on $a^{\prime} b^{\prime}$, but not on all three indices. Using $(63,64)$ in $(61)$ we can solve for $h_{\alpha i}{ }^{\beta j^{\prime}}$ in terms of the $Y \mathrm{~s}$ :

$$
\begin{aligned}
h_{a^{\prime}} & =-\frac{i}{7} Y_{a^{\prime}} \\
h_{a^{\prime} b^{\prime} c^{\prime}} & =-i Y_{a^{\prime} b^{\prime} c^{\prime}} \\
h_{a a^{\prime}} & =i Y_{a a^{\prime}} \\
h_{a a^{\prime} b^{\prime} c^{\prime}} & =-\frac{3 i}{5} Y_{a a^{\prime} b^{\prime} c^{\prime}} .
\end{aligned}
$$

In addition we find two constraints

$$
\begin{aligned}
Y_{a^{\prime} b^{\prime} ; c^{\prime}} & =0 \\
Y_{a a^{\prime} b^{\prime} c^{\prime} d^{\prime} ; e^{\prime}} & =0 .
\end{aligned}
$$

Finally, the fields $Y_{a a^{\prime} b^{\prime} ; c^{\prime}}, Y_{a^{\prime} b^{\prime} c^{\prime} d^{\prime} ; e^{\prime}}$ drop out of (61) and are therefore left undetermined.

\section{Solving the spinorial cohomology problem}

Let us recapitulate: the deformations of the supersymmetric M2 theory are parametrised by the object $\Sigma_{\alpha i}{ }^{\prime}$ in the $(1) \times(1001)$ of $\operatorname{Spin}(1,2) \times \operatorname{Spin}(8) . \Sigma_{\alpha i}{ }^{a^{\prime}}$ is not arbitrary, but has to satisfy the two constraints (65). In the language of section 4 , such a $\Sigma_{\alpha i} a^{\prime}$ determines an element of 
the spinorial cohomology group $H_{B^{\prime}}^{1}$. These constraints tell us that the projection of the spinor derivative of $\Sigma_{\alpha i}{ }^{\prime}$ onto the $(0) \times(1100) \oplus(2) \times(1002)$ part should vanish. This gives us the explicit form of the spinorial derivative $d_{s}$ for this case:

$$
\left.\left.d_{s}\left(\Sigma_{\alpha i} a^{\prime}\right) \rightarrow\left(\sigma^{a_{1}^{\prime} a_{2}^{\prime}}\right)^{i j} \epsilon^{\alpha \beta} \nabla_{\alpha i} \Sigma_{\beta j}^{b^{\prime}}\right|_{(1100)} \oplus\left(\sigma^{a_{1}^{\prime} \ldots a_{5}^{\prime}}\right)^{i j} \gamma_{a}^{\alpha \beta} \nabla_{\alpha i} \Sigma_{\beta j}^{b^{\prime}}\right|_{(1002)},
$$

where the bars denote the projections onto the indicated irreducible Spin(8) representations. In addition one has to take into account the field redefinitions. As explained in the previous section, these are given by the projection of the spinor derivative of $(\delta z)^{a^{\prime}}$ onto the $(1) \times(1001)$ part. Explicitly,

$$
\left.d_{s}(\delta z)^{a^{\prime}} \rightarrow \nabla_{\alpha i}(\delta z)^{a^{\prime}}\right|_{(1001)}
$$

Our strategy is to view $\Sigma_{\alpha i} a^{\prime},(\delta Z)^{a^{\prime}}$ as composite operators, given in terms of the world-volume fields $X_{a b}{ }^{c^{\prime}}, \Lambda_{a}{ }^{\alpha i^{\prime}}$. At any given order of $\ell$, one writes down the most general expressions for $\Sigma_{\alpha i}{ }^{a^{\prime}},(\delta z)^{a^{\prime}}$ allowed by dimensional analysis and representation theory. In determining the most general expressions at a given order in $\ell$ one can assume that the worldvolume fields obey the lowest-order equations. This is because $\Sigma_{\alpha i}^{a^{\prime}},(\delta z)^{a^{\prime}}$ are already of order $\beta$. In terms of irreducible representations of $\operatorname{Spin}(1,2) \times \operatorname{Spin}(8)$,

$$
\begin{aligned}
\Lambda_{a}{ }^{\alpha i^{\prime}} & \sim(3) \times(0010) \\
X_{a b}^{c^{\prime}} & \sim(4) \times(1000) \\
\nabla_{a} \Lambda_{b}{ }^{\alpha i^{\prime}} & \sim(5) \times(0010), \quad \text { up to terms of the form } \Lambda^{3} \\
\nabla_{a} X_{b c}{ }^{d^{\prime}} & \sim(6) \times(1000), \quad \text { up to terms of the form } X \Lambda^{2}
\end{aligned}
$$

\subsection{Spinorial cohomology at $\beta=\ell^{2}$}

By dimensional analysis $\Sigma_{\alpha i}{ }^{\prime}$ is schematically of the form ${ }^{3}$

$$
\Sigma \sim \nabla \Lambda \oplus X \Lambda \oplus \Lambda^{3}
$$

In terms of irreducible representations of $\operatorname{Spin}(1,2) \times \operatorname{Spin}(8)$,

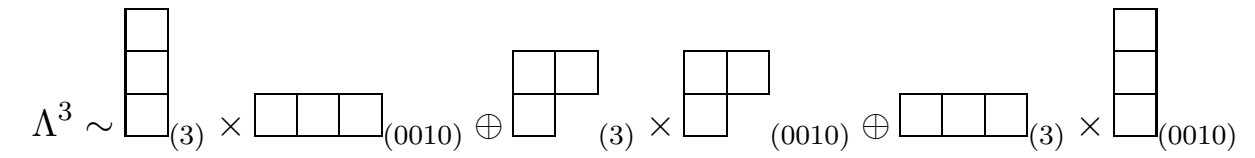

$$
\begin{aligned}
& \nabla \Lambda \sim(5) \times(0010) \\
& X \Lambda \sim(4) \times(1000) \otimes(3) \times(0010),
\end{aligned}
$$

where we have taken into account the fact that $\Lambda$ is anticommuting. One can verify that $(1) \times$ (1001) is not contained in the decomposition of the right-hand sides above and therefore there is no possible composite field $\Sigma_{\alpha i}{ }^{\prime}$ at this order in $\beta$. Consequently, the spinorial cohomology is trivial and there are no possible supersymmetric deformations of the theory at this order.

\footnotetext{
${ }^{3}$ In this equation and other similar schematic equations, the symbol $\nabla$ indicates the even covariant derivative $\nabla_{a}$
} 


\subsection{Spinorial cohomology at $\beta=\ell^{3}$}

In this section we shall show that $H_{B^{\prime}}^{1}($ phys $)=0$ at order $\beta=\ell^{3}$. By dimensional analysis $\Sigma_{\alpha i}{ }^{\prime}$ has the form

$$
\Sigma \sim X^{2} \Lambda \oplus \nabla^{2} \Lambda \oplus \nabla X \Lambda \oplus X \nabla \Lambda \oplus X \Lambda^{3} \oplus \Lambda^{2} \nabla \Lambda \oplus \Lambda^{5} .
$$

Analysing this in terms of representations of $\operatorname{Spin}(1,2) \times \operatorname{Spin}(8)$ one finds that only the boxed terms contain $(1) \times(1001)$. The three contributing terms are

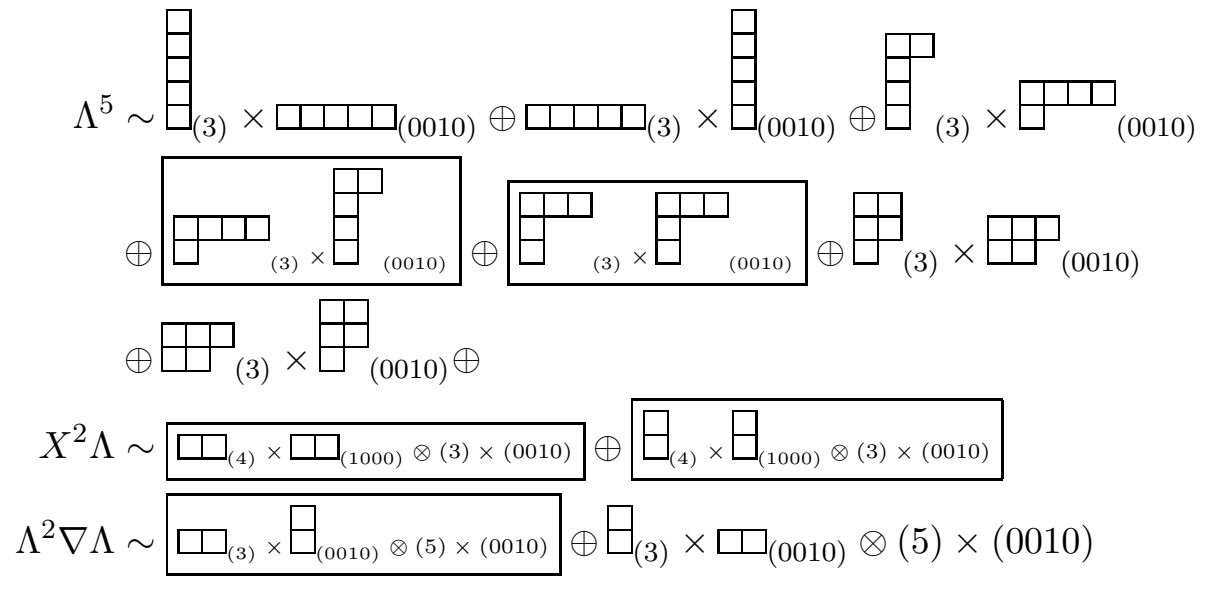

Analysing the contents of these products in terms of irreducible representations, we find that only the boxed expressions contain the representation of $\Sigma$, i.e. (1) $\times(1001)$. So at order $\ell^{3}$ a nontrivial $\Sigma$ must be built from these five terms and must satisfy the constraints (65).

We now identify the field redefinitions which can be used to remove some of these terms. Dimensional analysis restricts the field redefinitions $(\delta z)^{a^{\prime}}$ at order $\ell^{3}$ to be of the types $X^{2}, X \Lambda \Lambda$ and $\Lambda^{4}$. Decomposing these into irreducible representations one finds that that only $X \Lambda \Lambda$ contains a $\operatorname{Spin}(8)$ vector which is a $\operatorname{Spin}(1,2)$ scalar as required, and so the field redefinitions are given as:

$$
\Lambda^{2} X \sim\left(\square_{(3)} \times \square_{(0010)}\right) \otimes(4) \times(1000)
$$

This means that we can remove one of the five terms in $\Sigma$ by a field redefinition.

Next, we need to find the terms which contribute to the constraints (65). This can be done either by explicitly calculating the action of $d_{s}$ on all five terms, or by dimensional analysis and representation theory. The latter method reveals that, schematically

$$
Y \sim X \Lambda \nabla \Lambda, \Lambda^{2} \nabla X \text { and } \Lambda^{4} X
$$

Figure 6.2 illustrates how the field redefinitions generate contributions to $\Sigma$ and how $\Sigma$ generates contributions to the constraints. We can choose to remove the $\Lambda^{3} \nabla \Lambda$-type term, and are then 


\section{(1)}

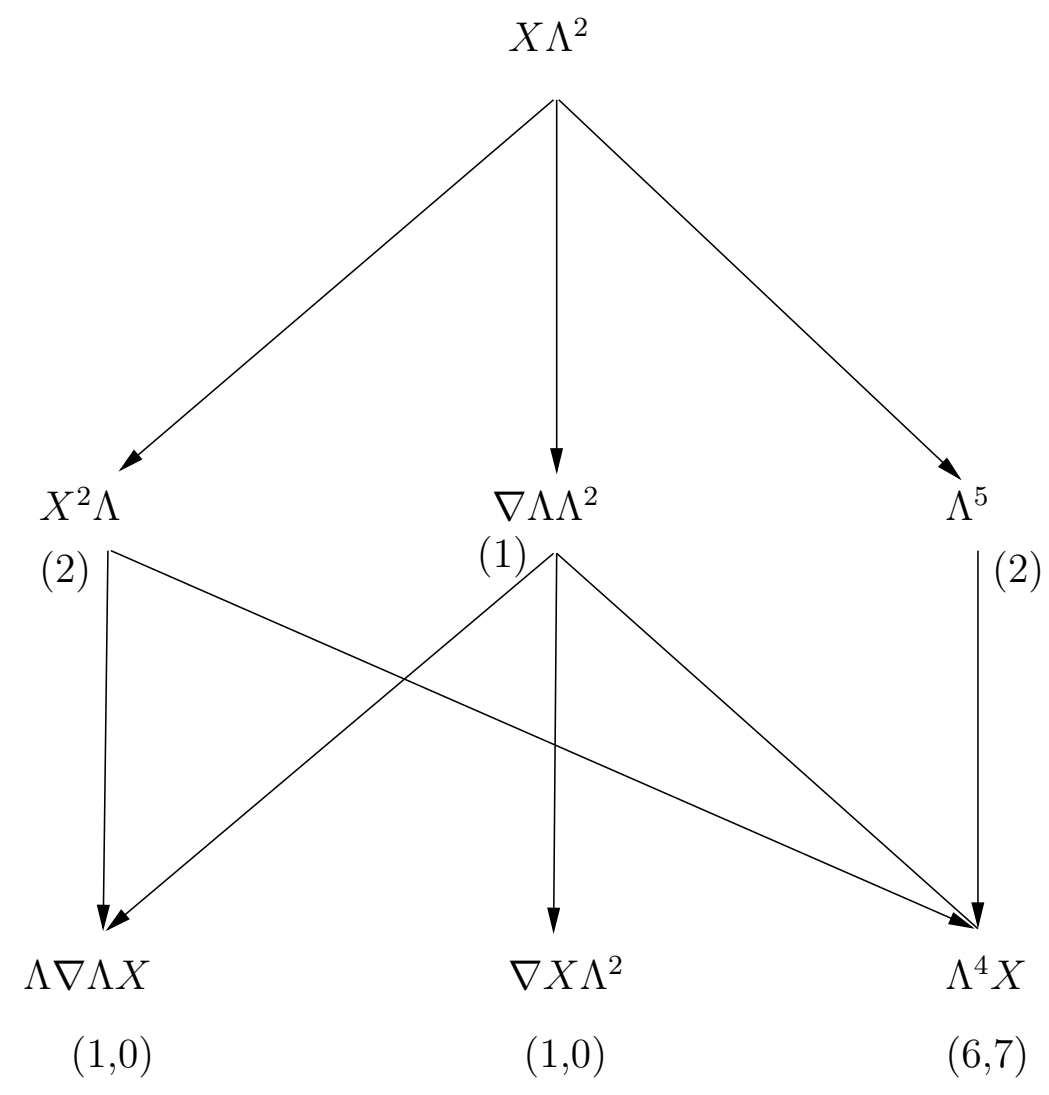

Figure 1: Spinorial cohomology at $\ell^{3}$. The three rows contain, schematically, all possible terms in $(\delta z)^{a^{\prime}}$ (field redefinitions), $\Sigma_{\alpha i} a^{\prime}$ (traceless part of $\psi$ ), $Y_{a^{\prime} b^{\prime} ; c^{\prime}}$ and $Y_{a a^{\prime} b^{\prime} c^{\prime} d^{\prime} ; e^{\prime}}$ (constraints). Multiplicities are denoted by the numbers in parentheses. In the third row the first number in

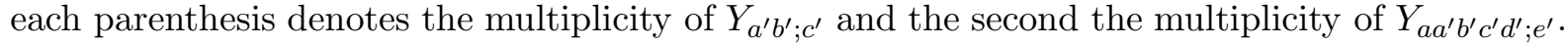
The arrows from the first to second, and second to third, rows indicate the action of $d_{s}$.

left with four terms that must not violate the constraints, i.e. contribute to the $(0) \times(1100)$ or $(2) \times(1002)$. To show that any linear combination of these terms, that is any content of $\psi$ at this order in $\ell$, would violate the constraints, we need to go into more detail. We break up the possible terms for $\Sigma$ and those for the constraints $Y$ into products of irreducible representations (which one can derive by dimensional analysis and representation theory as before). This is illustrated in figure 6.2. As indicated in the figure, after using the field redefinition, we are left with two different $X^{2} \Lambda$ and two different $\Lambda^{5}$ terms in $\Sigma$. We can choose a basis for the $X^{2} \Lambda$ such that only one of them contributes to the forbidden $\Lambda \nabla \Lambda X$ (arrows indicating its contributions to other representation in $Y$ are suppressed). The latter cannot be generated from either of the $\Lambda^{5}$ terms. Therefore, the first $X^{2} \Lambda$-term would violate the constraints and cannot be present. An explicit calculation then shows that the remaining $X^{2} \Lambda$ term contributes nontrivially to the $X \Lambda^{4}$ with a $(2,2)$ plethysm of the $\Lambda$. Again, neither of the $\Lambda^{5}$ terms can cancel this, and so the second $X^{2} \Lambda$ term must also be trivial (again, arrows indicating its contributions to other representations in $Y$ are suppressed). Finally, as the arrows in the figure indicate, the 


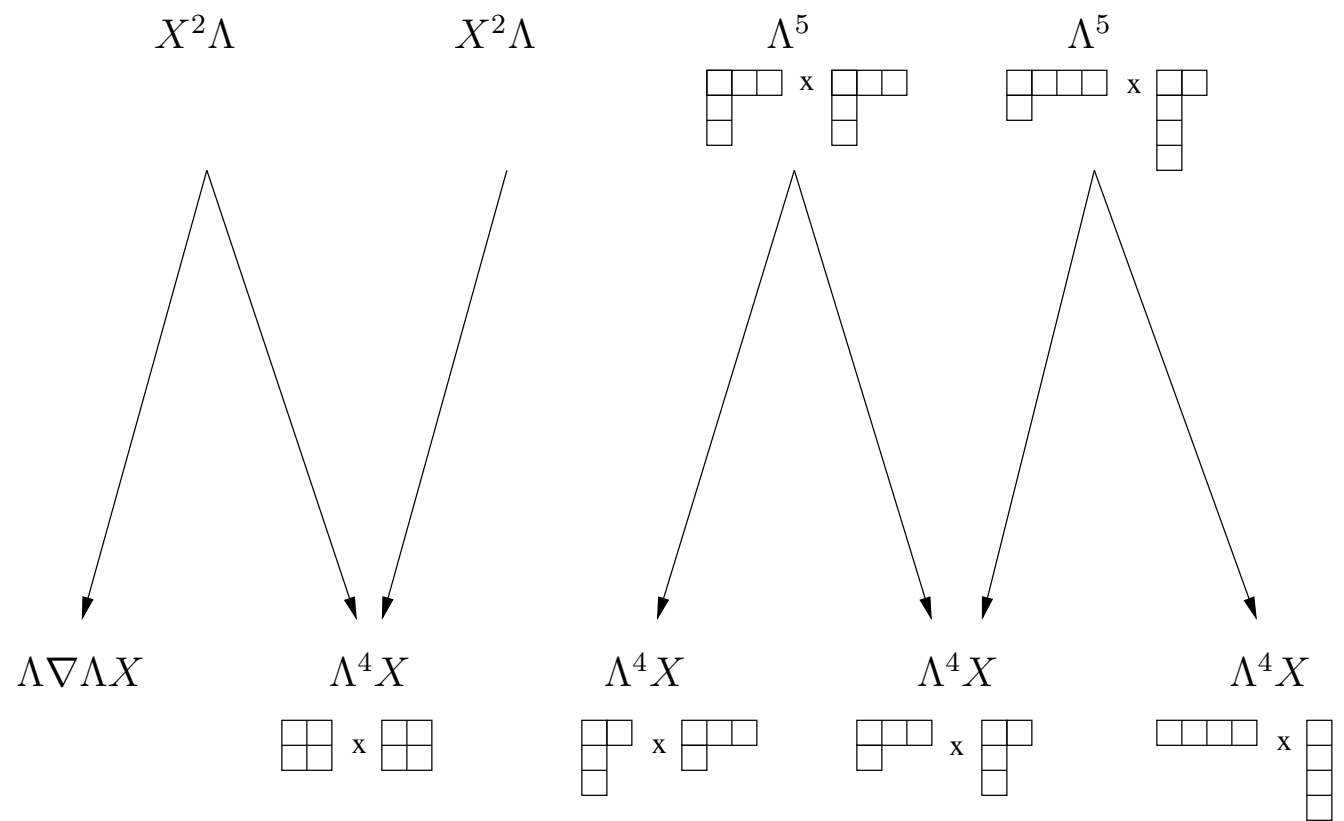

Figure 2: The first row represents possible terms for $\Sigma$ and the second row those for $Y$. The arrows indicate the action of $d_{s}$ (the spinorial derivative). Some arrows are suppressed. The products of Young-Tableaux represent plethysms of $\Lambda$, so that the left factor is a tableau of the (3) of $\operatorname{Spin}(1,2)$ and the right factor is a tableau of the (0010) of $\operatorname{Spin}(8)$.

$\Lambda^{5}$ terms cannot remove each other's contributions towards $Y$. This shows that there is no linear combination of the potential terms which obey the constraints.

\subsection{Spinorial cohomology at $\beta=\ell^{4}$}

By dimensional analysis $\Sigma_{\alpha i}{ }^{\prime}$ is, schematically,

$$
\begin{aligned}
\Sigma & \sim X^{3} \Lambda \oplus X \nabla X \Lambda \oplus X^{2} \nabla \Lambda \oplus X \nabla^{2} \Lambda \oplus \nabla^{2} X \Lambda \oplus \nabla X \nabla \Lambda \oplus \nabla^{3} \Lambda \\
& \oplus X^{2} \Lambda^{3} \oplus \nabla X \Lambda^{3} \oplus X \nabla \Lambda \Lambda^{2} \oplus \Lambda^{2} \nabla^{2} \Lambda \oplus(\nabla \Lambda)^{2} \Lambda \oplus X \Lambda^{5} \oplus \nabla \Lambda \Lambda^{4} \oplus \Lambda^{7}
\end{aligned}
$$

In terms of irreducible representations of $\operatorname{Spin}(1,2) \times \operatorname{Spin}(8)$, we can decompose the above contributions as

$$
\begin{aligned}
\Lambda^{7} & \sim 7(1) \times(1001) \oplus \ldots \\
\Lambda^{4} \nabla \Lambda & \sim 12(1) \times(1001) \oplus \ldots \\
X^{2} \Lambda^{3} & \sim 28(1) \times(1001) \oplus \ldots \\
X^{2} \nabla \Lambda & \sim 2(1) \times(1001) \oplus \ldots \\
\Lambda^{2} \nabla^{2} \Lambda & \sim 1(1) \times(1001) \oplus \ldots \\
(\nabla \Lambda)^{2} \Lambda & \sim 1(1) \times(1001) \oplus \ldots \\
X \nabla X \Lambda & \sim 4(1) \times(1001) \oplus \ldots
\end{aligned}
$$


In addition one can verify that there are no more contributions coming from the rest of the terms on the right-hand side of (69). There are therefore fifty-five possible terms.

We shall now make the assumption that there is a non-trivial cohomology element cubic in the fields. This is what we expect from string theory calculations in ten dimensions. In order to determine the cubic part $\left(\Sigma_{\alpha i}^{(c u b) a^{\prime}}\right)$ of the cohomology, we shall only require the explicit form of the cubic terms. These are eight in total,

$$
\begin{aligned}
& \Sigma_{\alpha i}^{(1) a^{\prime}}=\left.X^{b ; c a^{\prime}} \nabla_{b} X_{c}^{a b^{\prime}}\left(\sigma_{b^{\prime}} \Lambda_{a}\right)_{\alpha i}\right|_{(1001)} \\
& \Sigma_{\alpha i}^{(2) a^{\prime}}=\left.X^{b c b^{\prime}} \nabla_{b} X_{c}^{a a^{\prime}}\left(\sigma_{b^{\prime}} \Lambda_{a}\right)_{\alpha i}\right|_{(1001)} \\
& \Sigma_{\alpha i}^{(3) a^{\prime}}=\left.X^{b c a^{\prime}} \nabla_{b} X^{a d b^{\prime}}\left(\gamma_{c d} \otimes \sigma_{b^{\prime}} \Lambda_{a}\right)_{\alpha i}\right|_{(1001)} \\
& \Sigma_{\alpha i}^{(4) a^{\prime}}=\left.X^{b c b^{\prime}} \nabla_{b} X^{a d a^{\prime}}\left(\gamma_{c d} \otimes \sigma_{b^{\prime}} \Lambda_{a}\right)_{\alpha i}\right|_{(1001)} \\
& \Sigma_{\alpha i}^{(5) a^{\prime}}=\left.\left(\sigma_{b^{\prime}} \nabla_{b} \Lambda_{c}\right)_{\alpha i} X^{b d a^{\prime}} X_{d}^{c b^{\prime}}\right|_{(1001)} \\
& \Sigma_{\alpha i}^{(6) a^{\prime}}=\left.\left(\gamma^{b c} \otimes \sigma_{b^{\prime}} \nabla^{d} \Lambda^{e}\right)_{\alpha i} X_{b d}^{a^{\prime}} X_{c e}^{b^{\prime}}\right|_{(1001)} \\
& \Sigma_{\alpha i}^{(7) a^{\prime}}=\left.\left(\Lambda^{b} \gamma^{d} \otimes \sigma^{a^{\prime} b^{\prime}} \Lambda^{c}\right)\left(\sigma_{b^{\prime}} \nabla_{b} \nabla_{c} \Lambda_{d}\right)_{\alpha i}\right|_{(1001)} \\
& \Sigma_{\alpha i}^{(8) a^{\prime}}=\left.\left(\Lambda^{b} \gamma^{d} \otimes \sigma^{a^{\prime} b^{\prime}} \nabla_{b} \Lambda^{c}\right)\left(\sigma_{b^{\prime}} \nabla_{c} \Lambda_{d}\right)_{\alpha i}\right|_{(1001)}
\end{aligned}
$$

so that

$$
\Sigma_{\alpha i}^{(c u b) a^{\prime}}=\sum_{I=1}^{8} c^{I} \Sigma_{\alpha i}^{(I) a^{\prime}} .
$$

The coefficients $c^{I}$ will be determined, up to an overall factor, in the following.

Carrying out a similar analysis for the redefinitions $(\delta z)^{a^{\prime}}$ at this order in $\beta$ we conclude that the only possible contributions are of the form,

$$
\begin{aligned}
\Lambda^{4} X & \sim 5(0) \times(1000) \oplus \ldots \\
\Lambda^{2} \nabla X & \sim 1(0) \times(1000) \oplus \ldots \\
X \Lambda \nabla \Lambda & \sim 2(0) \times(1100) \oplus \ldots \\
X^{3} & \sim 1(0) \times(1100) \oplus \ldots
\end{aligned}
$$

There are nine terms in total, but we shall only need the explicit form of the following cubic terms,

$$
\begin{aligned}
& (\delta z)^{(1) a^{\prime}}=X^{b c a^{\prime}} X_{b d b^{\prime}} X_{c}{ }^{d b^{\prime}} \\
& (\delta z)^{(2) a^{\prime}}=\left(\Lambda^{b} \gamma^{d} \otimes \sigma^{a^{\prime} b^{\prime}} \Lambda^{c}\right) \nabla_{b} X_{c d b^{\prime}} \\
& (\delta z)^{(3) a^{\prime}}=\left(\Lambda^{b} \gamma^{d} \otimes \sigma^{a^{\prime} b^{\prime}} \nabla_{b} \Lambda^{c}\right) X_{c d b^{\prime}} \\
& (\delta z)^{(4) a^{\prime}}=\left(\Lambda^{d} \gamma^{b} \nabla^{c} \Lambda_{d}\right) X_{b c}{ }^{a^{\prime}} .
\end{aligned}
$$

Finally, we need to repeat the analysis for the constraints $Y_{a^{\prime} b^{\prime} ; c^{\prime}}$ and $Y_{a a^{\prime} b^{\prime} c^{\prime} d^{\prime} ; e^{\prime}}$. We find the 
following contributions,

$$
\begin{aligned}
X^{2} \nabla X & \sim 1(0) \times(1100) \oplus \ldots \\
\nabla X \nabla \Lambda \Lambda & \sim 1(0) \times(1100) \oplus \ldots \\
X \nabla^{2} \Lambda \Lambda & \sim 1(0) \times(1100) \oplus \ldots \\
X^{3} \Lambda^{2} & \sim 12(0) \times(1100) \oplus 13(2) \times(1002) \oplus \ldots \\
X \nabla \Lambda \Lambda^{3} & \sim 26(0) \times(1100) \oplus 38(2) \times(1002) \oplus \ldots \\
\nabla X \Lambda^{4} & \sim 6(0) \times(1100) \oplus 7(2) \times(1002) \oplus \ldots \\
X \Lambda^{6} & \sim 22(0) \times(1100) \oplus 35(2) \times(1002) \oplus \ldots
\end{aligned}
$$

We shall only need the explicit form of the following terms,

$$
\begin{aligned}
& Y^{(1) a^{\prime} b^{\prime} ; c^{\prime}}=\left.X^{b c a^{\prime}} X_{a}{ }^{d b^{\prime} \mid} \nabla_{b} X^{\left.a e \mid c^{\prime}\right]} \varepsilon_{c d e}\right|_{(1100)} \\
& Y^{(1) a^{\prime} b^{\prime} ; c^{\prime}}=\left.\left(\nabla^{b} \Lambda^{c} \sigma^{b^{\prime} c^{\prime}} \Lambda^{a}\right) \nabla_{b} X_{c a}{ }^{a^{\prime}}\right|_{(1100)} \\
& Y^{(1) a^{\prime} b^{\prime} ; c^{\prime}}=\left.\left(\nabla_{b} \nabla_{c} \Lambda_{a} \sigma^{b^{\prime} c^{\prime}} \Lambda^{a}\right) X^{b c a^{\prime}}\right|_{(1100)} .
\end{aligned}
$$

All the above is summarised in the following diagram. The coefficients $c^{I}$ in (70) are determined, up to an overall factor, as follows. First note that the redefinitions (71) can be used to eliminate $\Sigma_{\alpha i}^{(I) a^{\prime}} ; I=5 \ldots 8$. Indeed an explicit calculation gives

$$
\begin{aligned}
& d_{s}(\delta z)^{(2) a^{\prime}} \rightarrow \Sigma_{\alpha i}^{(7) a^{\prime}}+\ldots \\
& d_{s}(\delta z)^{(3) a^{\prime}} \rightarrow i \Sigma_{\alpha i}^{(8) a^{\prime}}+\ldots
\end{aligned}
$$

so that $(\delta z)^{(2,3) a^{\prime}}$ can be used to eliminate $\Sigma_{\alpha i}^{(7,8) a^{\prime}}$. Moreover,

$$
\begin{aligned}
& d_{s}(\delta Z)^{(1) a^{\prime}} \rightarrow 2 i \Sigma_{\alpha i}^{(5) a^{\prime}}+\ldots \\
& d_{s}(\delta Z)^{(4) a^{\prime}} \rightarrow-\frac{1}{2} \Sigma_{\alpha i}^{(5) a^{\prime}}+\frac{1}{2} \Sigma_{\alpha i}^{(6) a^{\prime}}+\ldots,
\end{aligned}
$$

and so $(\delta z)^{(1,4) a^{\prime}}$ can be used to eliminate $\Sigma_{\alpha i}^{(5,6) a^{\prime}}$. In the following we set

$$
c^{I}=0 ; \quad I=5 \ldots 8 .
$$

Next we evaluate the action of $d_{s}$ on the remaining cubic terms,

$$
\begin{aligned}
& d_{s} \Sigma_{\alpha i}^{(1) a^{\prime}} \rightarrow-4 i Y^{(3) a^{\prime} b^{\prime} ; c^{\prime}}+\ldots \\
& d_{s} \Sigma_{\alpha i}^{(2) a^{\prime}} \rightarrow-4 i Y^{(2) a^{\prime} b^{\prime} ; c^{\prime}}+\ldots \\
& d_{s} \Sigma_{\alpha i}^{(3) a^{\prime}} \rightarrow-16 i Y^{(1) a^{\prime} b^{\prime} ; c^{\prime}}-4 i Y^{(3) a^{\prime} b^{\prime} ; c^{\prime}}+\ldots \\
& d_{s} \Sigma_{\alpha i}^{(4) a^{\prime}} \rightarrow-32 i Y^{(1) a^{\prime} b^{\prime} ; c^{\prime}}+4 i Y^{(2) a^{\prime} b^{\prime} ; c^{\prime}}+\ldots,
\end{aligned}
$$

where the ellipses stand for terms with more than three fields. Demanding the vanishing of $d_{s}$ fixes the remaining coefficients to be

$$
\begin{aligned}
c^{2} & =\frac{1}{2} c^{1} \\
c^{3} & =-c^{1} \\
c^{4} & =\frac{1}{2} c^{1} .
\end{aligned}
$$




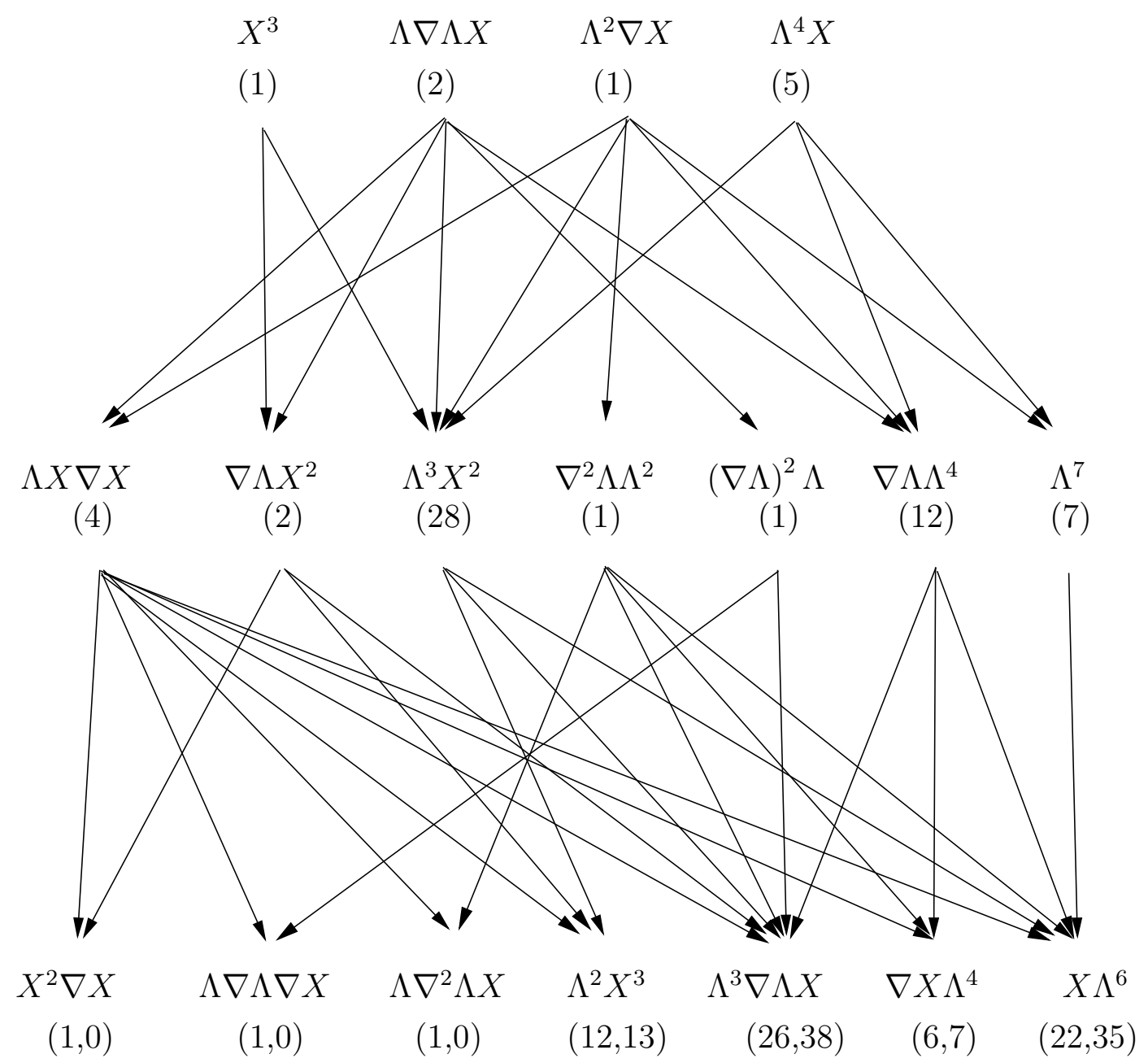

Figure 3: Spinorial cohomology at $\ell^{4}$.

We conclude that the part of the spinorial cohomology cubic in the fields at order $\ell^{4}$ is determined up to an overall factor to be represented by

$$
\begin{aligned}
\Sigma_{\alpha i}^{a^{\prime}=} & \left.X^{b c a^{\prime}} \nabla_{b} X_{c}^{a b^{\prime}}\left(\sigma_{b^{\prime}} \Lambda_{a}\right)_{\alpha i}\right|_{(1001)}+\left.\frac{1}{2} X^{b c b^{\prime}} \nabla_{b} X_{c}^{a a^{\prime}}\left(\sigma_{b^{\prime}} \Lambda_{a}\right)_{\alpha i}\right|_{(1001)} \\
& -\left.X^{b c a^{\prime}} \nabla_{b} X^{a d b^{\prime}}\left(\gamma_{c d} \otimes \sigma_{b^{\prime}} \Lambda_{a}\right)_{\alpha i}\right|_{(1001)} \\
& +\left.\frac{1}{2} X^{b c b^{\prime}} \nabla_{b} X^{a d a^{\prime}}\left(\gamma_{c d} \otimes \sigma_{b^{\prime}} \Lambda_{a}\right)_{\alpha i}\right|_{(1001)}
\end{aligned}
$$

\section{Action}

There are various ways of deriving the action corresponding to the deformed embedding constraint. One is to compute the modified equations of motion by solving the torsion constraints at dimensions one-half and one, and then to work back to the Lagrangian. This is a systematic approach, but is fairly tedious in practice. Another possibility is to use the action principle for 
branes. We shall discuss this below, but it turns out that one is required to solve another spinorial cohomology problem. In order to avoid this we shall find the key part of the Lagrangian up to quadratic order in fermions by brutal force. Nevertheless, the action principle does shed light on the structure of the component action.

\subsection{Action principle}

We recall that supersymmetric Lagrangians in $D$-dimensional spacetime can be constructed from closed superspace $D$-forms $[36,37]$. Given such a form, $L$, the spacetime action is

$$
S=\int \epsilon^{m_{1} \ldots m_{D}} L_{m_{1} \ldots m_{D}}(x, 0)
$$

Under an infinitesimal superspace diffeomorphism generated by a vector field $v, \delta L=\mathcal{L}_{v} L=$ $i_{v} d L+d\left(i_{v} L\right)=d\left(i_{v} L\right)$ because $L$ is closed. By evaluating this variation at $\theta=0$ on the spacetime part of the $D$-form one can see that the action (73) is invariant under spacetime diffeomorphisms and local supersymmetry transformations, these being identified as the leading components of the even and odd superspace diffeomorphisms.

In the context of branes one can construct a Lagrangian form starting from the (closed) WessZumino $(d+1)$-form $W$ on the super worldvolume, where $d$ is the dimension of the bosonic worldvolume. This can be written in terms of an explicit potential $Z, W=d Z$, but it can also be written in terms of a globally-defined $d$-form, $W=d K$. The $d$-form $L=K-Z$ is closed by construction. It is uniquely defined up to the exterior derivative of a $(d-1)$-form which is irrelevant in the action. When the standard embedding constraint $E_{\alpha} \underline{a}=0$ is imposed (in the

on-shell or Lagrangian off-shell cases) this procedure gives the Green-Schwarz action, including the Born-Infeld contribution in the case of $D$-branes [38]. At higher orders, it must still be possible to write $W$ as $d K$, but we shall also require Lagrangian forms satisfying $d L=0$.

For the M2-brane $W$ is the pull-back of the target space four-form $H$ on to the brane, $W=$ $f^{*} \underline{H}:=H$. The components of the pullback are (up to signs)

$$
H_{A B C D}=E_{D} \underline{\underline{D}} E_{C} \underline{\underline{C}} E_{B} \underline{\underline{B}} E_{A} \underline{\underline{A}} H_{\underline{A B C D}} .
$$

The only non-vanishing component of $\underline{H}$ in flat superspace is

$$
H_{\underline{a b}, \underline{\gamma} \underline{\delta}}=-i\left(\Gamma_{\underline{a b}}\right)_{\underline{\gamma} \underline{\delta}}
$$

To order $\beta$ we therefore have

dim. -1

$$
H_{\alpha \beta \gamma \delta}=0
$$

dim. $-1 / 2$

$$
H_{a \beta \gamma \delta}=0
$$

dim. 0

$$
H_{a b \gamma \delta}=-i\left(\Gamma_{a b}\right)_{\gamma \delta}-4 i \Lambda_{[a}^{\alpha^{\prime}}\left(\Gamma_{b] c^{\prime}}\right)_{\alpha^{\prime}(\gamma} \psi_{\delta}{ }^{c^{\prime}}
$$


dim. $1 / 2$

$$
H_{a b c \delta}=-3 i\left(\Gamma_{[a b \mid}\right)_{\gamma^{\prime} \delta^{\prime}} \Lambda_{\mid c]} \gamma^{\prime} h_{\delta}^{\delta^{\prime}}
$$

dim. 1

$$
H_{a b c d}=0
$$

We can now solve $H=d K$. In components,

$$
H_{A B C D}=4 \nabla_{[A} K_{B C D]}+6 T_{[A B \mid}^{F} K_{F \mid C D]}
$$

Note that for $\beta=0$, all the components of $K$ are zero, except for the highest-dimension component $K_{a b c}$. To order $\beta$ we have, taking (75-79) into account ,

dim. -1

$$
0=-\frac{3 i}{2}\left(\Gamma^{f}\right)_{(\alpha \beta \mid} K_{f \mid \gamma \delta)}+\nabla_{(\alpha} K_{\beta \gamma \delta)}
$$

This is solved by

$$
K_{\alpha \beta \gamma}=0 ; \quad K_{a \beta \gamma}=0 .
$$

dim. $-1 / 2$

$$
0=\left(\Gamma^{f}\right)_{(\beta \gamma \mid} K_{f a \mid \delta)}
$$

This implies

$$
K_{a b \gamma}=0
$$

dim. 0

$$
-i\left(\Gamma_{a b}\right)_{\gamma \delta}-4 i \Lambda_{[a} \alpha^{\prime}\left(\Gamma_{b] c^{\prime}}\right)_{\alpha^{\prime}(\gamma} \psi_{\delta}{ }^{c^{\prime}}=\left(-i\left(\Gamma^{e}\right)_{\gamma \delta}-2 i \Lambda^{e \alpha^{\prime}}\left(\Gamma_{c^{\prime}}\right)_{\alpha^{\prime}(\gamma} \psi_{\delta}{ }^{c^{\prime}}\right) K_{e a b}
$$

where we have taken into account the expression for $T_{\alpha \beta}{ }^{e}$ to order $\beta$, given in section three. The above equation is satisfied when

$$
K_{a b c}=\varepsilon_{a b c} .
$$

This is identical to the solution for $K_{A B C}$ in the $\beta=0$ case. This does not necessarily mean that there is no correction to the Green-Schwarz action because the induced Green-Schwarz metric may change. However, one can check that there is no such change to first order in $\beta$ although corrections of this type will arise at higher orders.

This means that the Lagrangian form we are looking for must satisfy $d L=0$, and, furthermore, that $L_{\alpha \beta \gamma}$ cannot be zero. Since the Lagrangian form can be changed by an exact form without affecting the action (73), it follows that this gives rise to a new spinorial cohomology problem. This is more complicated than the one treated in section six in that the representations involved are larger. On dimensional grounds, the order $\beta$ contribution to $L_{\alpha \beta \gamma}$ which is quartic in fields has the schematic form $\Lambda^{3} X$ but we have not attempted to compute it explicitly. However, it is useful to observe that this method of constructing actions tells us something about the structure of the component Lagrangian. From (73) we have

$$
\epsilon^{m n p} L_{m n p}=\epsilon^{m n p}\left(E_{p}{ }^{c} E_{n}{ }^{b} E_{m}{ }^{a} L_{a b c}+3 E_{p}{ }^{c} E_{n}{ }^{b} E_{m}{ }^{\alpha} L_{\alpha b c}+\ldots\right)
$$


The non-leading terms on the right-hand side all involve the induced worldvolume "gravitino" field $E_{m}{ }^{\alpha}$. This is related to $\partial_{m} \theta^{\underline{\alpha}}$ but it is not expressible in terms of $\Lambda$. The components $L_{A B C}$ will all be constructed from manifest tensors, namely $\Lambda$ and $X$; the gravitino terms reflect the fact that the Lagrangian is a density.

\subsection{Component approach}

In the component approach we write the action as $S=S_{0}+S_{1}$, where $S_{0}$ is the usual GreenSchwarz action for the supermembrane [31], and similarly for the worldvolume supersymmetry variations, so that at first order we have

$$
\delta_{1} S_{0}+\delta_{0} S_{1}=0
$$

We can ignore the first term if we calculate $S_{1}$ using the lowest-order field equations. The neglected terms correspond to field redefinitions. We are therefore looking for an on-shell invariant at order $\beta$.

From the discussion above we have seen that the first-order Lagrangian density $\mathcal{L}$ will have the form

$$
\mathcal{L}=\sqrt{-g} \hat{L}+\epsilon^{m n p}\left(3 E_{p}{ }^{c} E_{n}{ }^{b} E_{m}{ }^{\alpha} L_{\alpha b c}+\ldots\right)
$$

where $g$ is the determinant of the Green-Schwarz metric, $\hat{L}:=\epsilon^{a b c} L_{a b c}$ and where the right-hand side is understood to be evaluated at $\theta=0$. Under a worldvolume supersymmetry variation we find

$$
\begin{aligned}
\delta \mathcal{L}= & \partial_{m}\left(v^{\alpha} \mathcal{L}_{\alpha}^{m}\right) \\
= & \delta(\sqrt{-g}) \hat{L}+\sqrt{-g} \delta \hat{L} \\
& +\epsilon^{m n p}\left(3 E_{p}{ }^{c} E_{n}{ }^{b} E_{m}{ }^{\alpha} \delta L_{\alpha b c}+6 E_{p}{ }^{c}\left(\delta E_{n}{ }^{b}\right) E_{m}{ }^{\alpha} L_{\alpha b c}+3 E_{p}{ }^{c} E_{n}{ }^{b}\left(\delta E_{m}{ }^{\alpha}\right) L_{\alpha b c} \ldots\right)
\end{aligned}
$$

for some $\mathcal{L}_{\alpha}^{m}$. To quadratic order in fermions the terms represented by the dots in (82), which have two or more powers of $E_{m}{ }^{\alpha}$, can be disregarded. The schematic form of the Lagrangian terms are

$$
\begin{aligned}
\hat{L} & \sim X^{4}+\Lambda^{2} \nabla X^{2}+\mathcal{O}\left(\Lambda^{4}\right) \\
L_{\alpha b c} & \sim \Lambda X^{3}+\mathcal{O}\left(\Lambda^{3}\right)
\end{aligned}
$$

while

$$
\begin{aligned}
\delta E_{m}{ }^{a} & =i v^{\beta}\left(\Gamma^{a}\right)_{\beta \gamma} E_{m}{ }^{\gamma} \\
\delta E_{m}{ }^{\alpha} & =\nabla_{m} v^{\alpha}+v^{\beta} E_{m}{ }^{c} T_{\beta c}{ }^{\alpha} .
\end{aligned}
$$


We shall focus on the terms in the variation of $\mathcal{L}$ which are linear in fermions. In the variation of $E_{m}{ }^{\alpha}$ we can use the lowest-order expression for the induced torsion $T_{\beta c}{ }^{\alpha}$. As this is quadratic in $\Lambda$ the corresponding term in $\delta E_{m}{ }^{\alpha}$ can be ignored. The term involving the variation of the world-volume vielbein $E_{m}{ }^{a}$ can also be ignored as it leads to terms which are cubic in fermions. The terms which are linear in fermions involve either $\Lambda$ or $E_{m}{ }^{\alpha}$, and these must cancel separately. Thus we see that the term arising from the variation of the determinant of the metric multiplied by the $X^{4}$ term in $\hat{L}$ must be cancelled by the leading term coming from the variation of $L_{\alpha b c}$. The terms linear in $\Lambda$ arise from varying both the $\Lambda^{0}$ and $\Lambda^{2}$ terms in $\hat{L}$ and from the $\nabla v$ variation term in $E_{m}{ }^{\alpha}$ multiplied by $L_{\alpha b c}$. It therefore follows that the terms linear in $\Lambda$ in the variation of $\hat{L}$ will cancel up to a covariant divergence if the parameter $v^{\alpha}$ is taken to be covariantly constant. The terms involving the derivative of $v$ in the variation of $\hat{L}$ must cancel against similar terms arising from the variation of $E_{m}{ }^{\alpha}$. In fact, demanding that these terms cancel determines $L_{\alpha b c}$ at first order in $\Lambda$. We shall therefore concentrate on computing $\hat{L}$ up to quadratic order in the fermions. In what follows we shall refer to $\hat{L}$ as the Lagrangian.

It follows from the analysis in section six that, up to and including terms quadratic in the fermions, there is a unique supersymmetric invariant at order $l^{4}$. Indeed, we have shown that at order cubic in the fields there is a unique nontrivial element in the relevant spinorial cohomology group and this implies that there is a unique deformation of the equations of motion. We conclude that in the action there is a unique supersymmetric invariant at order quartic in the fields. In particular, there can be no terms in the action of order cubic, or less, in the fields. There are also no terms of the form $\Lambda^{2} X^{3}$ as can be seen by the fact that there is no scalar in the decomposition of the tensor product

$$
[(3) \times(0010)]^{2 \otimes_{a}} \otimes[(4) \times(1000)]^{3 \otimes_{s}},
$$

The bosonic part of the Lagrangian at $l^{4}$, is a linear combination of the following three $X^{4}$ terms

$$
\begin{aligned}
& t_{1}:=X_{a b}{ }^{a^{\prime}} X_{c d a^{\prime}} X^{a b b^{\prime}} X^{c d} b_{b^{\prime}} \\
& t_{2}:=X_{a b}{ }^{a^{\prime}} X_{c d}{ }^{b^{\prime}} X^{a b}{ }_{a^{\prime}} X^{c d} b_{b^{\prime}} \\
& t_{3}:=X_{a b}{ }^{a^{\prime}} X_{c d}{ }^{b^{\prime}} X^{a c}{ }_{a^{\prime}} X^{b d} b_{b^{\prime}}
\end{aligned}
$$

A fourth structure

$$
t_{4}:=X_{a b}{ }^{a^{\prime}} X_{c d a^{\prime}} X^{a c b^{\prime}} X^{b d} b^{\prime}
$$

can be shown to be linearly dependent

$$
t_{4}=t_{1}+\frac{1}{2} t_{2}-2 t_{3}
$$

by using the identity

$$
\left.4 X_{[a}^{\left[b \mid a^{\prime}\right.} X_{c]} \mid d\right] b^{\prime}=\left(\delta_{f}^{e} X^{g h a^{\prime}} X_{g h}^{b^{\prime}}-X_{f}^{g a^{\prime}} X_{g}^{e b^{\prime}}-X_{f}^{g b^{\prime}} X_{g}^{e a^{\prime}}\right) \varepsilon_{e a c} \varepsilon^{f b d} .
$$

At quadratic order in the fermions, the Lagrangian at $l^{4}$ contains terms of the form $\Lambda^{2} X \nabla X$ and $\Lambda \nabla \Lambda X^{2}$. A lengthy calculation, the details of which are given in appendix $\mathrm{B}$, yields the following result for $\hat{L}$ : 


$$
\begin{aligned}
\hat{L}= & \frac{1}{4} X_{a b} a^{\prime} X_{c d a^{\prime}} X^{a b b^{\prime}} X_{b^{\prime}}^{c d}-\frac{1}{4} X_{a b} a^{\prime} X_{c d} b^{\prime} X_{a^{\prime}}^{a c} X_{b^{\prime}}^{b d} \\
& +i\left(\Lambda_{a} \gamma_{b} \nabla_{c} \Lambda_{d}\right) X^{a b a^{\prime}} X_{a^{\prime}}^{c d}-\frac{2 i}{3}\left(\Lambda^{f} \gamma_{a} \otimes \sigma^{a^{\prime} b^{\prime}} \nabla_{f} \Lambda_{b}\right) X_{a^{\prime}}^{a e} X_{e b^{\prime}}^{b} \\
& -\frac{i}{3}\left(\Lambda_{a} \gamma_{b} \otimes \sigma^{a^{\prime} b^{\prime}} \nabla_{c} \Lambda_{d}\right) X_{a^{\prime}}^{a b} X_{b^{\prime}}^{c d}+\mathcal{O}\left(\Lambda^{4}\right) .
\end{aligned}
$$

The Lagrangian above is determined up to a total derivative and up to terms vanishing by virtue of the lowest-order equations of motion. The latter can always by removed by appropriate field redefinitions.

We shall now compare our result to that of [2]. In this paper the bosonic CP-even part of the $\mathcal{O}\left(l^{4}\right)$ Lagrangian of a D- $p$ brane was determined to be

$$
\frac{1}{16}\left(\left(R_{T}\right)_{a b c d}\left(R_{T}\right)^{a b c d}-2\left(R_{T}\right)_{a b}\left(R_{T}\right)^{a b}-\left(R_{N}\right)_{a b c^{\prime} d^{\prime}}\left(R_{N}\right)^{a b c^{\prime} d^{\prime}}+2 \bar{R}_{a^{\prime} b^{\prime}} \bar{R}^{a^{\prime} b^{\prime}}\right),
$$

see equation (2.14) of that reference. In the above we have omitted an overall multiplicative constant involving $\alpha^{\prime}$ and the string coupling.

Let us view (92) as the world-volume Lagrangian of a D2-brane and consider its lift to eleven dimensions. The various curvatures in (92) are given, in the notation of the present paper and for flat target-space, by

$$
\begin{aligned}
\left(R_{T}\right)_{a b c d} & =X_{a c a^{\prime}} X_{b d}{ }^{a^{\prime}}-X_{a d a^{\prime}} X_{b c}{ }^{a^{\prime}} \\
\left(R_{N}\right)_{a b c^{\prime} d^{\prime}} & =X_{a e c^{\prime}} X^{e}{ }_{b d^{\prime}}-X_{b e c^{\prime}} X^{e}{ }_{a d^{\prime}} \\
\left(R_{T}\right)_{a b} & =-X_{a e} a^{a^{\prime}} X_{b a^{\prime}} \\
\bar{R}_{a^{\prime} b^{\prime}} & =X_{a b a^{\prime}} X^{a b}{ }_{b^{\prime}} .
\end{aligned}
$$

Substituting (93) into (92) on gets precisely the bosonic part of (91).

The Lagrangian (92) was determined, as is the case with (91), modulo total derivatives ${ }^{4}$ and terms which vanish by virtue of the lowest-order equations of motion. In [3] the investigation of these ambiguities was considered, see equation (5.4) therein. It was shown that there is a total of five ambiguous terms, two of which vanish by virtue of the lowest-order equations of motion and therefore cannot be determined perturbatively. Two of the other three terms vanish for flat target-space, whereas the remaining third term vanishes by virtue of equation (89) of the present paper.

\section{Kappa symmetry}

Although we have not used the modified worldvolume supersymmetry transformations in determining the Lagrangian, it is perhaps of interest to see how the usual $\kappa$-symmetry variations are altered. To lowest order the $\kappa$-symmetry transformations were derived, starting from worldvolume supersymmetry transformations, in equations (9-14). These will be amended when we

\footnotetext{
${ }^{4}$ the coefficient of the Gauss-Bonnet term was fixed to zero in [2] by an indirect argument.
} 
deform the embedding condition. In general, a worldvolume supersymmetry transformation gives the following transformations

$$
\begin{aligned}
& \delta z^{\underline{a}}=v^{\alpha} E_{\alpha} \underline{a} \\
& \delta z^{\underline{\alpha}}=v^{\alpha} E_{\alpha} \underline{\alpha} .
\end{aligned}
$$

We can convert the second of these into $\kappa$-symmetry form as before:

$$
\delta z^{\underline{\alpha}}=\kappa^{\underline{\beta}} P_{\underline{\beta}}^{\underline{\alpha}}=\frac{1}{2} \kappa^{\underline{\beta}}(1+\Gamma)_{\underline{\beta}}^{\underline{\alpha}} .
$$

where $\kappa^{\underline{\alpha}}=v^{\alpha} E_{\alpha} \underline{\alpha}$ and

$$
P_{\underline{\alpha}}^{\underline{\beta}}=\left(E^{-1}\right)_{\underline{\alpha}}^{\gamma} E_{\gamma} \underline{\beta}
$$

where the inverse refers to inverting the square matrix formed from $\left(E_{\alpha} \underline{\alpha}, E_{\alpha^{\prime}} \underline{\alpha}\right)$. Explicitly

$$
P_{\underline{\alpha}} \underline{\beta}=\left(u^{-1}\right)_{\underline{\alpha}}^{\gamma} u_{\gamma} \underline{\beta}+\left(u^{-1}\right)_{\underline{\alpha}}^{\gamma} h_{\gamma}^{\delta^{\prime}} u_{\delta^{\prime}} \underline{\beta}
$$

The first term here has the same structure as in the undeformed theory. The bosonic projection of $\delta z \underline{M}$ can be written

$$
\delta z^{\underline{a}}=\kappa^{\underline{\alpha}} \Psi_{\underline{\alpha}}^{\underline{a}}=\delta z^{\underline{\alpha}} \Psi_{\underline{\alpha}}^{\underline{a}}
$$

where

$$
\begin{aligned}
\Psi_{\underline{\alpha}}^{\underline{a}} & =\left(E^{-1}\right)_{\underline{\alpha}}^{\gamma} E_{\gamma} \underline{a} \\
& =\left(E^{-1}\right)_{\underline{\alpha}}^{\gamma} \psi_{\gamma}{ }^{a^{\prime}} u_{a^{\prime}} \underline{a}
\end{aligned}
$$

satisfies $\Psi=P \Psi$.

In the undeformed theory the matrix $\Gamma$ is given by

$$
\Gamma=-\frac{1}{6} \epsilon^{a b c} u_{a}{ }^{\underline{a}} u_{b} \underline{\underline{b}} u_{c} \underline{\underline{c}} \Gamma_{\underline{a b c}} .
$$

This can easily be seen to be the same as

$$
\Gamma_{\underline{\alpha}}^{\underline{\beta}}=\left(u^{-1}\right)_{\underline{\alpha}}^{\gamma} u_{\gamma}^{\underline{\beta}}-\left(u^{-1}\right)_{\underline{\alpha}}^{\gamma^{\prime}} u_{\gamma^{\prime}} \underline{\beta}
$$

In the deformed theory we can write 


$$
\Gamma=\Gamma_{0}+\frac{1}{2} u^{-1}\left(1+\Gamma_{0}\right) h\left(1-\Gamma_{0}\right) u
$$

where $\Gamma_{0}$ has the same form as in (101) and (102) and where $h$ is regarded as a $32 \times 32$ matrix satisfying $h=P_{0} h Q_{0}$, with $P_{0}=1 / 2\left(1+\Gamma_{0}\right), Q=1 / 2\left(1-\Gamma_{0}\right)$.

The formulae above are valid for an arbitrary deformation $\psi$. To obtain the order $\beta$ term we merely have to substitute into the expressions the explicit forms for $\psi$ and $h$.

\section{Conclusions}

In this paper we have discussed the first correction to the dynamics of the M2-brane in the superembedding formalism which we have shown to occur at order $\ell^{4}$. We have seen that the allowed modifications of the constraints are specified by an element of a certain spinorial cohomology group. There is a unique solution cubic in the fields and we believe that there are no other independent terms at quartic or higher orders. In principle one could continue this analysis for higher powers of $\ell$. We would expect to find both corrections induced by iterating the first correction and new cohomological terms. However, the computations are difficult, even at $\ell^{4}$.

The formalism developed here could be applied straightforwardly to other branes such as the M5-brane and D-branes. Indeed, we could obtain the equivalent terms in the D2-brane action by dimensional reduction. In particular, this would shed some light on the $\kappa$-symmetric effective action including $\partial^{4} F^{4}$ terms. Another extension of the formalism would be to include non-trivial supergravity backgrounds. In $D=11$ it is expected that the first correction to supergravity occurs at $\ell^{6}$, so that this complication could be ignored. It should therefore be relatively straightforward to compute the corrections at the same order, which involve the background supergravity fields. In the case of the M5-brane these results could again be dimensionally reduced to ten dimensions, either to give results for the NS5-brane, or the D4-brane. In the latter case

one would expect to see some sign of the $\hat{A}$-genus terms discussed in [39, 40,41]. In principle, we should therefore be able to obtain some information about the supersymmetrisation of these terms.

\section{Acknowledgements}

This work was supported in part by EU contracts HPRN-2000-00122 (which includes Queen Mary, London as a subcontractor) and HPRN-CT-2000-00148 and PPARC grants PPA/G/S/ 1998/00613 and PPA/G/O/2000/00451. SFK thanks the German National Merit Foundation for financial support. UL acknowledges support in part by VR grant 650-1998368. 


\section{Appendix A}

\section{Notation}

The following index conventions are used: plain (underlined) indices refer to worldsurface and target space quantities respectively and primed indices refer to normal spaces; indices from the beginning of the alphabet refer to preferred bases, indices from the middle of the alphabet to coordinate bases; Latin (Greek) indices are used for even (odd) indices, while capital indices run over the whole space. Thus a worldsurface preferred basis index could be $A=(a, \alpha)$ while a target space coordinate index could be $\underline{M}=(\underline{m}, \underline{\mu})$. Tensor quantities with indices are not underlined, but if the indices are omitted or if a target space tensor is projected on some of its indices we underline the tensor.

For the spinor indices a two step notation is used. Initially the target space index $\underline{\alpha}$, running from 1 to 32 is split in two, $\underline{\alpha} \rightarrow\left(\alpha, \alpha^{\prime}\right)$, where both the worldsurface index $\alpha$ and the normal index $\alpha^{\prime}$ run from 1 to 16 . For some purposes this is adequate but sometimes one wishes to recognise explicitly that these indices transform under $\operatorname{Spin}(1,2) \times \operatorname{Spin}(8)$. We set

$$
\begin{aligned}
\psi^{\alpha} & \rightarrow \psi^{\alpha i} \\
\psi^{\alpha^{\prime}} & \rightarrow \psi^{\alpha i^{\prime}}
\end{aligned}
$$

where the spinor index on the right takes on two values while $i$ and $i^{\prime}$ both run from 1 to 8 . We use space-favoured metrics throughout, $\eta_{a b}=(-1,+1, \ldots,+1)$, and the spacetime $\epsilon$-tensors have $\epsilon^{0123 \cdots}=+1$.

We use the following representation of the $D=11 \Gamma$-matrices:

$$
\begin{aligned}
\Gamma^{a} & =\gamma^{a} \otimes \gamma_{9} \\
\Gamma^{a^{\prime}} & =1 \otimes \gamma^{a^{\prime}}
\end{aligned}
$$

where the $d=3 \gamma$-matrices are $2 \times 2$ and the $d^{\prime}=8 \gamma$-matrices are $16 \times 16$. The charge conjugation matrix is

$$
C=\epsilon \otimes \gamma_{9}
$$

In indices

$$
\begin{aligned}
\left(\Gamma^{a}\right)_{\underline{\alpha}} \underline{\beta} & =\left(\gamma^{a}\right)_{\alpha}^{\beta}\left(\begin{array}{cc}
\delta_{i}^{j} & 0 \\
0 & -\delta_{i^{\prime}} j^{\prime}
\end{array}\right) \\
\left(\Gamma^{a^{\prime}}\right)_{\underline{\alpha}}^{\underline{\beta}} & =\delta_{\alpha}{ }^{\beta}\left(\begin{array}{cc}
0 & \left(\sigma^{a^{\prime}}\right)_{i}^{j^{\prime}} \\
\left(\tilde{\sigma}^{a^{\prime}}\right)_{i^{\prime}}{ }^{j} & 0
\end{array}\right)
\end{aligned}
$$

and

$$
C_{\underline{\alpha \beta}}=\epsilon_{\alpha \beta}\left(\begin{array}{cc}
\delta_{i j} & 0 \\
0 & -\delta_{i^{\prime} j^{\prime}}
\end{array}\right)
$$

where the $8 \times 8 \sigma$-matrices are related to the eight-dimensional $\gamma$-matrices by

$$
\gamma^{a^{\prime}}=\left(\begin{array}{cc}
0 & \left(\sigma^{a^{\prime}}\right)_{i j^{\prime}} \\
\left(\tilde{\sigma}^{\alpha^{\prime}}\right)_{i^{\prime} j} & 0
\end{array}\right)
$$


Eleven-dimensional spinor indices are raised or lowered according to the rule

$$
\psi^{\underline{\alpha}}=C \underline{\alpha \underline{\beta}} \psi_{\underline{\beta}} \leftrightarrow \psi_{\underline{\alpha}}=\psi \underline{\underline{\beta}} C_{\underline{\beta} \underline{\alpha}}
$$

where $C$ with upper indices is the same matrix as $C$ with lower indices. Three-dimensional spinor indices are similarly raised or lowered using $\epsilon$. Eight-dimensional indices, whether spinor or vector, are raised and lowered with the standard Euclidean metric. The $\Gamma$-matrices with lowered indices are

$$
\begin{aligned}
\left(\Gamma^{a}\right)_{\underline{\alpha \beta}} & =\left(\gamma^{a}\right)_{\alpha \beta}\left(\begin{array}{cc}
\delta_{i j} & 0 \\
0 & \delta_{i^{\prime} j^{\prime}}
\end{array}\right) \\
\left(\Gamma^{a^{\prime}}\right)_{\underline{\alpha \beta}} & =\epsilon_{\alpha \beta}\left(\begin{array}{cc}
0 & -\left(\sigma^{a^{\prime}}\right)_{i j^{\prime}} \\
\left(\tilde{\sigma}^{a^{\prime}}\right)_{i^{\prime} j} & 0
\end{array}\right)
\end{aligned}
$$

It is straightforward to decompose any of the eleven-dimensional $\Gamma$-matrices with multi-vector indices in this way. We give the two-index $\Gamma$-matrices as they are used most in the text:

$$
\begin{aligned}
\left(\Gamma^{a b}\right)_{\underline{\alpha \beta}} & =\left(\gamma^{a b}\right)_{\alpha \beta}\left(\begin{array}{cc}
\delta_{i j} & 0 \\
0 & \delta_{i^{\prime} j^{\prime}}
\end{array}\right) \\
\left(\Gamma^{a b^{\prime}}\right)_{\underline{\alpha \beta}} & =-\left(\gamma^{a}\right)_{\alpha \beta}\left(\begin{array}{cc}
0 & -\left(\sigma^{b^{\prime}}\right)_{i j^{\prime}} \\
\left(\tilde{\sigma}^{b^{\prime}}\right)_{i^{\prime} j} & 0
\end{array}\right) \\
\left(\Gamma^{a^{\prime} b^{\prime}}\right)_{\underline{\alpha \beta}} & =\epsilon_{\alpha \beta}\left(\begin{array}{cc}
\left(\sigma^{a^{\prime} b^{\prime}}\right)_{i j^{\prime}} & 0 \\
0 & -\left(\tilde{\sigma}^{a^{\prime} b^{\prime}}\right)_{i^{\prime} j}
\end{array}\right)
\end{aligned}
$$

The three-dimensional $\gamma$-matrices are real, symmetric with lowered indices, and satisfy

$$
\gamma^{a} \gamma^{b}=\eta^{a b}+\gamma^{a b}
$$

where

$$
\gamma^{a b}=\epsilon^{a b c} \gamma_{c} \leftrightarrow \gamma_{a}=-\frac{1}{2} \epsilon_{a b c} \gamma^{b c}
$$

For the eight-dimensional $\sigma$-matrices one may take

$$
\begin{aligned}
& \sigma^{a^{\prime}}=\left(1, i \tau_{r}\right) \\
& \tilde{\sigma}^{a^{\prime}}=\left(1,-i \tau_{r}\right)
\end{aligned}
$$

where $r=1, \ldots 7$, and where the $\tau_{r}$ are seven-dimensional Dirac matrices which are purely imaginary and antisymmetric. The matrices $\sigma^{a^{\prime} b^{\prime} c^{\prime} d^{\prime}}$ and $\tilde{\sigma}^{a^{\prime} b^{\prime} c^{\prime} d^{\prime}}$ are symmetric, the former being self-dual, the latter anti-self-dual, $\sigma^{a^{\prime} b^{\prime}}$ and $\tilde{\sigma}^{a^{\prime} b^{\prime}}$ are antisymmetric.

\section{Group theoretic conventions}

Consider a Lie group $G$ and a $G$-module $V$. The $n$-th tensor product $V^{\otimes n}$ admits a decomposition

$$
\sum_{R} V_{R} \times R
$$

under $G \times S_{n}$, where $R$ runs over all irreducible representations of the symmetric group $S_{n}$ and $V_{R}$ is a $G$-module. As is well known, the irreducible representations of $S_{n}$ can be parametrised 
by partitions of $n$ or, equivalently, by the associated Young diagrams. If $R$ is associated to the partition $\lambda$ of $n, V_{R}$ is the plethysm of $V$ with respect to $\lambda$.

In this paper, we are using a notation referring to the product group $\operatorname{Spin}(1,2) \times \operatorname{Spin}(8)$.

The highest weight of the standard module (i.e. of the vector representation) of Spin(8) is given by (1000) on the basis of fundamental weights. A two-form is (0100) and a three-form is (0011). The self-dual and anti-self-dual four-forms are represented by (0002), (0020) respectively. The chiral and anti-chiral spinors are (0001), (0010). Moreover, the highest weight of the $k$ form-spinor is given by the sum of the highest weight of the $k$-form and the highest weight of the spinor. Recall that by a $k$-form-spinor we refer to the projection onto the irreducible (gamma-traceless) part. For example (1001) is a chiral vector-spinor, (0110) is an anti-chiral two-form-spinor, etc. We can also consider the projection onto the highest-weight representation of the plethysm associated to the partition $[2, \ldots, 2,1, \ldots, 1]$ of $n+k$, with $k$ entries equal to 2 and $n-k$ entries equal to 1 . This is one way to view the irreducible $(n, k)$-tensors of section 5 . For example, a (2,1)-tensor is a two-form and a one-form and is represented by (1100). A (3,1)tensor is a three-form and a one-form and is represented by (1011), etc. The highest-weights of irreducible (gamma-traceless) ( $n, k)$-tensor-spinors are represented in the obvious way. For example (1101) is the highest weight of a $(2,1)$-tensor-(chiral) spinor. This discussion generalizes straightforwardly to representations corresponding to more general partitions.

In the case of $\operatorname{Spin}(1,2)$ the situation is simpler, in that the representation denoted by $(n)$ is of dimension $n+1$. Hence, the spinor representation is denoted by (1), the vector by (2), the vector spinor by (3) and so on.

\section{Appendix B}

In this appendix we give some details of the derivation of the action in section seven. At quadratic order in the fermions the Lagrangian at $\ell^{4}$ contains terms of the form $\Lambda^{2} X \nabla X$ and $\Lambda \nabla \Lambda X^{2}$. We have the following structures:

$\Lambda^{2} X \nabla X$ :

$$
\begin{aligned}
& u_{1}:=\left(\Lambda^{f} \gamma_{a} \otimes \sigma^{a^{\prime} b^{\prime}} \Lambda_{f}\right) X_{c d a^{\prime}} \nabla^{a} X_{b^{\prime}}^{c d} \\
& u_{2}:=\left(\Lambda_{a} \gamma_{b} \otimes \sigma^{a^{\prime} b^{\prime}} \Lambda_{c}\right) X_{f a^{\prime}}^{a} \nabla^{f} X^{b c} b^{\prime} \\
& u_{3}:=\left(\Lambda_{a} \gamma_{b} \Lambda_{c}\right) X^{a}{ }_{f a^{\prime}} \nabla^{f} X^{b c a^{\prime}} \\
& u_{4}:=\left(\Lambda_{a} \gamma_{b} \otimes \sigma^{a^{\prime} b^{\prime}} \Lambda_{c}\right) X_{f a^{\prime}}^{b} \nabla^{f} X^{a c}{ }_{b^{\prime}}
\end{aligned}
$$

$\Lambda \nabla \Lambda X^{2}:$

$$
\begin{aligned}
& s_{1}:=\left(\Lambda^{f} \gamma_{a} \nabla_{f} \Lambda_{b}\right) X^{a e a^{\prime}} X_{e a^{\prime}}^{b} \\
& s_{2}:=\left(\Lambda_{a} \gamma_{b} \nabla_{c} \Lambda_{d}\right) X^{a b a^{\prime}} X_{a^{\prime}}^{c d} \\
& s_{3}:=\left(\Lambda^{f} \gamma_{a} \otimes \sigma^{a^{\prime} b^{\prime}} \nabla_{f} \Lambda_{b}\right) X^{a e}{ }_{a^{\prime}} X^{b}{ }_{e b^{\prime}} \\
& s_{4}:=\left(\Lambda_{a} \gamma_{b} \otimes \sigma^{a^{\prime} b^{\prime}} \nabla_{c} \Lambda_{d}\right) X^{a b}{ }_{a^{\prime}} X^{c d}{ }_{b^{\prime}} \\
& s_{5}:=\left(\Lambda_{a} \gamma_{b} \otimes \sigma^{a^{\prime} b^{\prime}} \nabla_{c} \Lambda_{d}\right) X_{a^{\prime}}^{a c} X^{b d} b_{b^{\prime}} \\
& s_{6}:=\left(\Lambda_{a} \gamma_{b} \nabla_{c} \Lambda_{d}\right) X^{a c a^{\prime}} X_{a^{\prime}}^{b d} .
\end{aligned}
$$


Not all of the above are independent. Only three of the $u$ and four of the $s$-terms are, as can be seen by counting the number of scalars in the decomposition of the tensor products

$$
[(3) \times(0010)]^{2 \otimes_{a}} \otimes[(4) \times(1000)] \otimes[(6) \times(1000)]
$$

and

$$
[(3) \times(0010)] \otimes[(5) \times(0010)] \otimes[(4) \times(1000)]^{2 \otimes_{s}} .
$$

Indeed on has

$$
\begin{aligned}
& 0=u_{2}-u_{4}-\frac{1}{2} u_{1} \\
& 0=s_{6}-s_{2}+s_{1} \\
& 0=3 s_{5}-s_{4}+s_{3} .
\end{aligned}
$$

The first line of (120) is derived using the identity

$$
\varepsilon_{e g h}\left(\Lambda^{g} \gamma^{h} \otimes \sigma^{a^{\prime} b^{\prime}} \Lambda_{c}\right)=\frac{1}{2} \varepsilon_{d e c}\left(\Lambda^{f} \gamma^{d} \otimes \sigma^{a^{\prime} b^{\prime}} \Lambda_{f}\right)
$$

The second line of (120) can be shown by taking (90) into account. Finally, to derive the third line of (120) one notes that

$$
0=\left(\Lambda_{e} \gamma^{[a e} \gamma_{b} \otimes \sigma_{a^{\prime} b^{\prime}} \nabla_{c} \Lambda_{d}\right) X_{a}^{c \mid a^{\prime}} X^{\mid b] d b^{\prime}}
$$

In addition one has to take into account the fact that some terms are related to each other by integration by parts. Indeed, up to total derivatives, one has the following relations

$$
\begin{aligned}
& u_{1}=2 s_{3} \\
& u_{2}=s_{4}-s_{5} \\
& u_{2}=u_{4}+s_{3} \\
& u_{3}=s_{2}-s_{6} \\
& u_{3}=s_{1} \\
& u_{4}=2 s_{5} \\
& u_{4}=u_{2}-s_{3} .
\end{aligned}
$$

Only six of the equations $(120,123)$ above are independent. It is perhaps interesting to note that the group-theoretic relations (120) are all implied by the partial integration equations (123). The solution may be given in terms of four independent variables which we take to be $s_{1-4}$,

$$
\begin{aligned}
u_{1} & =2 s_{3} \\
u_{2} & =\frac{1}{3} s_{3}+\frac{2}{3} s_{4} \\
u_{3} & =s_{1} \\
u_{4} & =-\frac{2}{3} s_{3}+\frac{2}{3} s_{4} \\
s_{5} & =-\frac{1}{3} s_{3}+\frac{1}{3} s_{4} \\
s_{6} & =-s_{1}+s_{2} .
\end{aligned}
$$


Therefore, the lagrangian at $l^{4}$ can be written as

$$
\hat{L}=\sum_{i=1}^{3} A_{i} t_{i}+\sum_{i=1}^{4} B_{i} s_{i}+\mathcal{O}\left(\Lambda^{4}\right),
$$

where the constant coefficients $A, B$ are determined by the requirement that the supersymmetric variation of $\hat{L}$ be a total derivative, up to $\nabla v$ terms.

At linear order in the fermions, the supersymmetric variation of the correction to the Lagrangian, igoniring the $\nabla v$ terms, consists of terms of the form $\nabla \Lambda X^{3}, \Lambda \nabla X X^{2}$. Explicitly, one can write down the following structures (suppressing spinor indices and the supersymmetry parameter $v$ ) $\nabla \Lambda X^{3}$ :

$$
\begin{aligned}
& w_{1}:=\left(\gamma_{a b} \otimes \sigma^{a^{\prime} b^{\prime} c^{\prime}} \nabla_{c} \Lambda_{d}\right) X_{e a^{\prime}}^{a} X_{b^{\prime}}^{e b} X^{c d}{ }_{c^{\prime}} \\
& w_{2}:=\left(\gamma_{a b} \otimes \sigma^{a^{\prime}} \nabla_{c} \Lambda_{d}\right) X_{e a^{\prime}}^{a} X_{b^{\prime}}^{e b} X^{c d b^{\prime}} \\
& w_{3}:=\left(\gamma_{a b} \otimes \sigma^{a^{\prime}} \nabla_{c} \Lambda_{d}\right) X^{a c}{ }_{a^{\prime}} X^{e b}{ }_{b^{\prime}} X_{e}{ }^{d b^{\prime}} \\
& w_{4}:=\left(\sigma^{a^{\prime}} \nabla_{a} \Lambda_{b}\right) X_{e a^{\prime}}^{a} X^{e g}{ }_{b^{\prime}} X_{g}^{b b^{\prime}} \\
& w_{5}:=\left(\sigma^{a^{\prime}} \nabla_{a} \Lambda_{b}\right) X^{a b b^{\prime}} X^{e g}{ }_{a^{\prime}} X_{e g b^{\prime}} \\
& w_{6}:=\left(\sigma^{a^{\prime}} \nabla_{a} \Lambda_{b}\right) X_{a^{\prime}}^{a b} X^{e g}{ }_{b^{\prime}} X_{e g} b^{b^{\prime}} \\
& w_{7}:=\left(\gamma_{a b} \otimes \sigma^{a^{\prime}} \nabla_{c} \Lambda_{d}\right) X^{a c}{ }_{b^{\prime}} X^{e b}{ }_{a^{\prime}} X_{e}{ }^{d b^{\prime}} \\
& w_{8}:=\left(\gamma_{a b} \otimes \sigma^{a^{\prime}} \nabla_{c} \Lambda_{d}\right) X_{b^{\prime}}^{a c} X_{a^{\prime}}^{e d} X_{e}^{b b^{\prime}} \\
& w_{9}:=\left(\sigma^{a^{\prime}} \nabla_{a} \Lambda_{b}\right) X_{e b^{\prime}}^{a} X^{e g}{ }_{a^{\prime}} X_{g}^{b b^{\prime}} \\
& w_{10}:=\left(\gamma_{a b} \otimes \sigma^{a^{\prime} b^{\prime} c^{\prime}} \nabla_{c} \Lambda_{d}\right) X_{a^{\prime}}^{c b} X_{e b^{\prime}}^{a} X^{e d}{ }_{c^{\prime}}
\end{aligned}
$$


$\Lambda \nabla X X^{2}:$

$$
\begin{aligned}
& z_{1}:=\left(\sigma^{a^{\prime}} \Lambda^{a}\right) \nabla_{a} X_{b c a^{\prime}} X^{b d b^{\prime}} X_{d b^{\prime}}^{c} \\
& z_{2}:=\left(\gamma^{a b} \otimes \sigma^{a^{\prime}} \Lambda^{c}\right) \nabla_{d} X_{b c a^{\prime}} X_{a e b^{\prime}} X^{d e b^{\prime}} \\
& z_{3}:=\left(\sigma^{a^{\prime}} \Lambda_{a}\right) \nabla_{b} X_{c d a^{\prime}} X^{a b b^{\prime}} X^{c d}{ }_{b^{\prime}} \\
& z_{4}:=\left(\gamma^{a b} \otimes \sigma^{a^{\prime}} \Lambda^{c}\right) \nabla_{b} X_{d e a^{\prime}} X_{c}{ }^{d b^{\prime}} X^{e}{ }_{a b^{\prime}} \\
& z_{5}:=\left(\sigma^{a^{\prime}} \Lambda^{a}\right) \nabla_{a} X_{b c}{ }^{b^{\prime}} X^{b d}{ }_{a^{\prime}} X^{c}{ }_{d b^{\prime}} \\
& z_{6}:=\left(\gamma^{a b} \otimes \sigma^{a^{\prime}} \Lambda^{c}\right) \nabla_{d} X_{b c b^{\prime}} X_{a e a^{\prime}} X^{d e b^{\prime}} \\
& z_{7}:=\left(\gamma^{a b} \otimes \sigma^{a^{\prime}} \Lambda^{c}\right) \nabla_{b} X_{d e b^{\prime}} X_{a c a^{\prime}} X^{d e b^{\prime}} \\
& z_{8}:=\left(\sigma^{a^{\prime}} \Lambda_{a}\right) \nabla_{b} X_{c d b^{\prime}} X^{a b a^{\prime}} X^{c d b^{\prime}} \\
& z_{9}:=\left(\gamma^{a b} \otimes \sigma^{a^{\prime}} \Lambda^{c}\right) \nabla_{d} X_{b c b^{\prime}} X_{a}^{e b^{\prime}} X^{d}{ }_{e a^{\prime}} \\
& z_{10}:=\left(\gamma^{a b} \otimes \sigma^{a^{\prime}} \Lambda^{c}\right) \nabla_{c} X_{d e}{ }^{b^{\prime}} X^{d}{ }_{a a^{\prime}} X^{e}{ }_{b b^{\prime}} \\
& z_{11}:=\left(\gamma^{a b} \otimes \sigma^{a^{\prime}} \Lambda^{c}\right) \nabla_{b} X_{d e} b^{b^{\prime}} X_{a c b^{\prime}} X^{d e} a^{\prime} \\
& z_{12}:=\left(\gamma^{a b} \otimes \sigma^{a^{\prime} b^{\prime} c^{\prime}} \Lambda^{c}\right) \nabla_{d} X_{b c a^{\prime}} X_{a e b^{\prime}} X^{d e}{ }_{c^{\prime}} \\
& z_{13}:=\left(\gamma^{a b} \otimes \sigma^{a^{\prime} b^{\prime} c^{\prime}} \Lambda^{c}\right) \nabla_{c} X_{d e a^{\prime}} X_{a b^{\prime}}^{d} X_{b c^{\prime}}^{e} \\
& z_{14}:=\left(\gamma^{a b} \otimes \sigma^{a^{\prime} b^{\prime} c^{\prime}} \Lambda^{c}\right) \nabla_{b} X_{d e a^{\prime}} X_{a c b^{\prime}} X^{d e} c_{c^{\prime}} \\
& z_{15}:=\left(\gamma^{a b} \otimes \sigma^{a^{\prime}} \Lambda^{c}\right) \nabla_{b} X_{d e b^{\prime}} X_{a e a^{\prime}} X_{c}{ }^{d b^{\prime}} \\
& z_{16}:=\left(\gamma^{a b} \otimes \sigma^{a^{\prime}} \Lambda^{c}\right) \nabla_{b} X^{d e b^{\prime}} X_{a e b^{\prime}} X_{c d a^{\prime}} \\
& z_{17}:=\left(\gamma^{a b} \otimes \sigma^{a^{\prime} b^{\prime} c^{\prime}} \Lambda^{c}\right) \nabla_{b} X^{d e}{ }_{a^{\prime}} X_{a e b^{\prime}} X_{c d c^{\prime}} \\
& z_{18}:=\left(\sigma^{a^{\prime}} \Lambda_{a}\right) \nabla_{b} X_{c d b^{\prime}} X^{a b b^{\prime}} X^{c d a^{\prime}} \\
& z_{19}:=\left(\gamma^{a b} \otimes \sigma^{a^{\prime}} \Lambda^{c}\right) \nabla_{b} X_{d e a^{\prime}} X^{d e b^{\prime}} X_{a c b^{\prime}} \\
& z_{20}:=\left(\sigma^{a^{\prime} b^{\prime} c^{\prime}} \Lambda_{a}\right) \nabla_{b} X_{c d a^{\prime}} X^{a b}{ }_{b^{\prime}} X^{c d}{ }_{c^{\prime}} .
\end{aligned}
$$

As before, not all of these structures are linearly independent. Only six of the $w$ and fourteen of the $z$-terms are, as one can see by counting the number of spinors $[(1) \times(0001)]$ in the decomposition of the tensor products

$$
[(5) \times(0010)] \otimes[(4) \times(1000)]^{3 \otimes_{s}}
$$

and

$$
[(3) \times(0010)] \otimes[(6) \times(1000)] \otimes[(4) \times(1000)]^{2 \otimes_{s}} .
$$

Indeed, taking into account (90) and the identity

$$
\gamma_{[a b} \Lambda_{c]}=0
$$

one can derive the following relations

$$
\begin{aligned}
w_{7} & =-w_{2}+w_{3}-w_{4}+\frac{1}{2} w_{6} \\
w_{8} & =w_{2}+w_{7} \\
w_{9} & =-2 w_{4}+w_{5}+\frac{1}{2} w_{6} \\
w_{10} & =\frac{1}{2} w_{1}
\end{aligned}
$$


and

$$
\begin{aligned}
z_{15}+z_{16} & =2 z_{5}-z_{6}+z_{7}-z_{9}+z_{11} \\
z_{15}-z_{16} & =z_{10} \\
z_{17} & =\frac{1}{2} z_{13} \\
z_{19} & =-z_{1}+z_{2}+z_{4} \\
z_{18} & =-z_{5}+z_{8}+z_{9}+z_{10}-z_{11}+z_{15} \\
2 z_{20} & =z_{12}-z_{13}-z_{14}-z_{17} .
\end{aligned}
$$

The last two lines in equation (132) above are derived by noting that

$$
\left(\gamma^{[e a} \sigma_{a^{\prime}} \Lambda_{e}\right) \nabla_{b} X_{c}^{d \mid b^{\prime}} X_{a}^{b a^{\prime}} X^{\mid c]} d b^{\prime}=0
$$

and

$$
\left(\gamma^{[e a \mid} \sigma^{a^{\prime} b^{\prime} c^{\prime}} \Lambda_{e}\right) \nabla_{b} X_{c}{ }^{|d|}{ }_{a^{\prime}} X_{a}{ }^{b} b^{\prime} X^{\mid c]} d c^{\prime}=0 .
$$

In addition one has to allow for integrations by parts. Up to total derivatives, one has the following relations

$$
\begin{aligned}
w_{1} & =2 z_{17} \\
w_{2} & =z_{4}-z_{15} \\
w_{3} & =z_{2}-z_{7} \\
w_{4} & =-z_{1}-z_{8} \\
w_{5} & =-z_{3}-z_{18} \\
w_{6} & =-2 z_{8} \\
w_{7} & =z_{6}-z_{19} \\
w_{8} & =z_{9}-z_{11} \\
w_{9} & =-z_{3}-z_{5} \\
w_{10} & =-z_{12}-z_{14}
\end{aligned}
$$


and

$$
\begin{aligned}
z_{1} & =-2 z_{5} \\
z_{2} & =z_{6}+z_{10} \\
z_{2} & =w_{4}-z_{16} \\
z_{4} & =w_{9}-z_{9} \\
z_{4} & =-w_{7}+z_{10} \\
z_{5} & =-w_{4}-z_{18} \\
z_{6} & =w_{4}-z_{15} \\
z_{7} & =\frac{1}{2} w_{6} \\
z_{10} & =-w_{3}-z_{15} \\
z_{11} & =w_{5}-z_{19} \\
z_{12} & =z_{17} \\
z_{13} & =2 z_{12} \\
z_{13} & =w_{10}+z_{17} \\
z_{16} & =-w_{8} \\
z_{20} & =0 .
\end{aligned}
$$

Equations (131, 132, 135, 136) above define an overdetermined system of thiry-five equations for thirty unknowns. It turns out however, that only twenty-four of the equations are linearly independent. Therefore all $w$ and $z$-terms are expressible in terms of six independent structures, which we can take to be $z_{1-4}, z_{7}, z_{12}$. Explicitly,

$$
\begin{aligned}
w_{1} & =2 z_{12} \\
w_{2} & =\frac{1}{2} z_{1}+z_{2}+z_{4}-z_{7} \\
w_{3} & =z_{2}-z_{7} \\
w_{4} & =-z_{1}+z_{7} \\
w_{5} & =-\frac{3}{2} z_{1}-z_{3}+z_{7} \\
w_{6} & =2 z_{7} \\
w_{7} & =\frac{1}{2} z_{1}-z_{4} \\
w_{8} & =z_{1}+z_{2}-z_{7} \\
w_{9} & =\frac{1}{2} z_{1}-z_{3} \\
w_{10} & =z_{12}
\end{aligned}
$$


and

$$
\begin{aligned}
z_{5} & =-\frac{1}{2} z_{1} \\
z_{6} & =-\frac{1}{2} z_{1}+z_{2} \\
z_{8} & =-z_{7} \\
z_{9} & =\frac{1}{2} z_{1}-z_{3}-z_{4} \\
z_{10} & =\frac{1}{2} z_{1} \\
z_{11} & =-\frac{1}{2} z_{1}-z_{2}-z_{3}-z_{4}+z_{7} \\
z_{13} & =2 z_{12} \\
z_{14} & =-2 z_{12} \\
z_{15} & =-\frac{1}{2} z_{1}-z_{2}+z_{7} \\
z_{16} & =-z_{1}-z_{2}+z_{7} \\
z_{17} & =z_{12} \\
z_{18} & =\frac{3}{2} z_{1}-z_{7} \\
z_{19} & =-z_{1}+z_{2}+z_{4} \\
z_{20} & =0
\end{aligned}
$$

Again we note that the group-theoretic relations $(131,132)$ are all implied by the partial integration equations $(135,136)$.

Next we compute the supersymmetric variation of the lagrangian (125). One finds, up to total derivatives and terms containing $\nabla v$,

$$
\begin{aligned}
\delta t_{1} & =-4 i w_{5} \\
\delta t_{2} & =-4 i w_{6} \\
\delta t_{3} & =-4 i w_{4} \\
\delta s_{1} & =\frac{1}{2}\left(w_{3}+w_{4}-z_{1}+z_{2}\right) \\
\delta s_{2} & =\frac{1}{2}\left(w_{2}+w_{5}-z_{3}+z_{19}\right) \\
\delta s_{3} & =\frac{1}{2}\left(w_{4}-w_{7}+w_{8}-w_{9}-w_{10}+z_{6}-z_{9}-z_{12}\right) \\
\delta s_{4} & =\frac{1}{2}\left(w_{1}+w_{2}-w_{5}+w_{6}+z_{7}-z_{8}-z_{11}-z_{14}+z_{18}\right) .
\end{aligned}
$$

Using $(137,138)$ it is straightforward to see that the vanishing of the variation of the lagrangian 
implies the following relations for the coefficients in (125),

$$
\begin{aligned}
& A_{1}=\frac{3 i}{4} B_{4} \\
& A_{2}=0 \\
& A_{3}=-\frac{3 i}{4} B_{4} \\
& B_{1}=0 \\
& B_{2}=-3 B_{4} \\
& B_{3}=2 B_{4} .
\end{aligned}
$$

These results lead to the Lagrangian (91).

\section{References}

[1] O. D. Andreev and A. A. Tseytlin, "Partition Function Representation For The Open Superstring Effective Action: Cancellation Of Mobius Infinities And Derivative Corrections To Born-Infeld Lagrangian," Nucl. Phys. B 311 (1988) 205.

[2] C. P. Bachas, P. Bain and M. B. Green, "Curvature terms in D-brane actions and their M-theory origin," JHEP 9905 (1999) 011 [arXiv:hep-th/9903210].

[3] A. Fotopoulos, "On $\alpha^{2}$ corrections to the D-brane action for non-geodesic world-volume embeddings," JHEP 0109 (2001) 005 [arXiv:hep-th/0104146].

[4] N. Wyllard, "Derivative corrections to D-brane actions with constant background fields," Nucl. Phys. B 598 (2001) 247 [arXiv:hep-th/0008125].

[5] A. Bilal, "Higher-derivative corrections to the non-abelian Born-Infeld action," Nucl. Phys. B 618 (2001) 21 [arXiv:hep-th/0106062].

[6] N. Wyllard, "Derivative corrections to the D-brane Born-Infeld action: Non-geodesic embeddings and the Seiberg-Witten map," JHEP 0108 (2001) 027 [arXiv:hep-th/0107185].

[7] P. Koerber and A. Sevrin, "The non-abelian D-brane effective action through order $\alpha^{4}$," JHEP 0210 (2002) 046 [arXiv:hep-th/0208044].

[8] U. Lindstrom, M. Rocek and P. van Nieuwenhuizen, "A Weyl Invariant Rigid String," Phys. Lett. B 199 (1987) 219.

[9] U. Lindstrom and M. Rocek, "Bosonic And Spinning Weyl Invariant Rigid Strings," Phys. Lett. B 201 (1988) 63.

[10] A. Collinucci, M. de Roo and M. G. Eenink, "Derivative corrections in 10-dimensional super-Maxwell theory," JHEP 0301 (2003) 039 [arXiv:hep-th/0212012].

[11] J. M. Drummond, P. J. Heslop, P. S. Howe and S. F. Kerstan, "Integral invariants in N = 4 SYM and the effective action for coincident D-branes," [arXiv:hep-th/0305202]. 
[12] E. A. Ivanov and A. A. Kapustnikov, "Gauge Covariant Wess-Zumino Actions For Super P-Branes In Superspace," Int. J. Mod. Phys. A 7 (1992) 2153.

[13] J. P. Gauntlett, “A kappa symmetry calculus for superparticles," Phys. Lett. B 272 (1991) 25 [arXiv:hep-th/9109039].

[14] E. A. Ivanov and A. A. Kapustnikov, "Towards A Tensor Calculus For Kappa Supersymmetry," Phys. Lett. B 267 (1991) 175.

[15] T. Curtright and P. van Nieuwenhuizen, "Supersprings," Nucl. Phys. B 294 (1987) 125.

[16] D. P. Sorokin, "Coincident (super)-Dp-branes of codimension one," JHEP 0108 (2001) 022 [arXiv:hep-th/0106212].

[17] S. Panda and D. Sorokin, "Supersymmetric and kappa-invariant coincident D0-branes," JHEP 0302 (2003) 055 [arXiv:hep-th/0301065].

[18] P. S. Howe and U. Lindstrom, "Kappa-symmetric higher derivative terms in brane actions," Class. Quant. Grav. 19 (2002) 2813 [arXiv:hep-th/0111036].

[19] J. M. Drummond, P. S. Howe and U. Lindstrom, "Kappa-symmetric non-Abelian BornInfeld actions in three dimensions," Class. Quant. Grav. 19 (2002) 6477 [arXiv:hepth/0206148].

[20] D. Sorokin, V. Tkach and D.V. Volkov, "Superparticles, twistors and Siegel symmetry," Mod. Phys. Lett. A4 (1989) 901; D. Sorokin, V. Tkach, D.V. Volkov and A. Zheltukhin, "From the superparticle Siegel symmetry to the spinning particle proper time supersymmetry," Phys. Lett. B216 (1989) 302; D.V. Volkov and A. Zheltukhin, "Extension of the Penrose representation and its use to describe supersymmetric models," Sov. Phys. JETP Lett. 48 (1988) 63-66.

[21] D. Sorokin, "Superbranes and superembeddings," Phys. Report 239 (2000) 1.

[22] P. S. Howe and E. Sezgin, "Superbranes," Phys. Lett. B 390 (1997) 133 [arXiv:hepth/9607227].

[23] P. S. Howe and E. Sezgin, "D = 11, p = 5," Phys. Lett. B 394 (1997) 62 [arXiv:hepth/9611008].

[24] M. Cederwall, B. E. Nilsson and D. Tsimpis, "The structure of maximally supersymmetric Yang-Mills theory: Constraining higher-order corrections," JHEP 0106 (2001) 034 [arXiv:hep-th/0102009].

[25] M. Cederwall, B. E. Nilsson and D. Tsimpis, "D = 10 super-Yang-Mills at $O\left(\alpha^{\prime 2}\right)$," JHEP 0107 (2001) 042 [arXiv:hep-th/0104236].

[26] M. Cederwall, B. E. Nilsson and D. Tsimpis, "Spinorial cohomology and maximally supersymmetric theories," JHEP 0202 (2002) 009 [arXiv:hep-th/0110069]. 
[27] M. Cederwall, B. E. Nilsson and D. Tsimpis, "Spinorial cohomology of abelian d = 10 super-Yang-Mills at $O\left(\alpha^{\prime 3}\right)$," JHEP 0211 (2002) 023 [arXiv:hep-th/0205165].

[28] P. S. Howe and D. Tsimpis, "On higher-order corrections in M theory," [arXiv:hepth/0305129].

[29] S. J. Gates and H. Nishino, "D = 2 Superfield Supergravity, Local (Supersymmetry) ${ }^{2}$ And Nonlinear Sigma Models," Class. Quant. Grav. 3 (1986) 391.

[30] I. A. Bandos, D. P. Sorokin, M. Tonin, P. Pasti and D. V. Volkov, "Superstrings and supermembranes in the doubly supersymmetric geometrical approach," Nucl. Phys. B 446 (1995) 79 [arXiv:hep-th/9501113].

[31] E. Bergshoeff, E. Sezgin and P. K. Townsend, "Supermembranes And Eleven-Dimensional Supergravity," Phys. Lett. B 189 (1987) 75.

[32] N. Berkovits, "Super-Poincare covariant quantization of the superstring," JHEP 0004 (2000) 018 [arXiv:hep-th/0001035].

[33] N. Berkovits, "Covariant quantization of the superparticle using pure spinors," JHEP 0109 (2001) 016 [arXiv:hep-th/0105050].

[34] P. S. Howe, "Pure Spinors Lines In Superspace And Ten-Dimensional Supersymmetric Theories," Phys. Lett. B 258 (1991) 141 [Addendum-ibid. B 259 (1991) 511].

[35] P. S. Howe, "Pure spinors, function superspaces and supergravity theories in ten-dimensions and eleven-dimensions," Phys. Lett. B 273 (1991) 90.

[36] R. D'Auria, P. Fre, P. K. Townsend and P. van Nieuwenhuizen, "Invariance Of Actions, Rheonomy And The New Minimal N=1 Supergravity In The Group Manifold Approach," Annals Phys. 155 (1984) 423.

[37] S.J. Gates, M. Grisaru, M. Knutt-Wehlau and W. Siegel, "Component actions from curved superspace: normal coordinates and ectoplasm," Phys.Lett. B421 (1998) 203-210, [arXiv:hep-th/9711151].

[38] P.S. Howe, O. Raetzel and E. Sezgin, "On brane actions and superembeddings," JHEP 9808 (1998) 011, [arXiv:hep-th/9804051].

[39] M. B. Green, J. A. Harvey and G. W. Moore, "I-brane inflow and anomalous couplings on D-branes," Class. Quant. Grav. 14 (1997) 47 [arXiv:hep-th/9605033].

[40] Y. K. Cheung and Z. Yin, "Anomalies, branes, and currents," Nucl. Phys. B 517 (1998) 69 [arXiv:hep-th/9710206].

[41] R. Minasian and G.W. Moore, "K theory and Ramond-Ramond charge," JHEP 9711 (1997) 002 [arXiv:hep-th/9710230]. 